\title{
Treating linear molecules in calculations of rotation-vibration spectra
}

Cite as: J. Chem. Phys. 153, 154106 (2020); https://doi.org/10.1063/5.0019546

Submitted: 23 June 2020 . Accepted: 30 September 2020 . Published Online: 16 October 2020

Sergei N. Yurchenko (D), and Thomas M. Mellor (D)
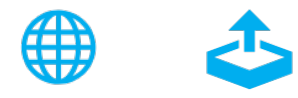

View Online

Export Citation

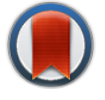

CrossMark

\section{ARTICLES YOU MAY BE INTERESTED IN}

Ground and excited state first-order properties in many-body expanded full configuration interaction theory

The Journal of Chemical Physics 153, 154107 (2020); https://doi.org/10.1063/5.0024791

Information theoretical and thermodynamic view of the excited-state density functional theory of Coulomb systems

The Journal of Chemical Physics 153, 154103 (2020); https://doi.org/10.1063/5.0015611

Core excitations with excited state mean field and perturbation theory

The Journal of Chemical Physics 153, 154102 (2020); https://doi.org/10.1063/5.0020595

\section{Meet the Next Generation of Quantum Analyzers And Join the Launch Event on November 17th}

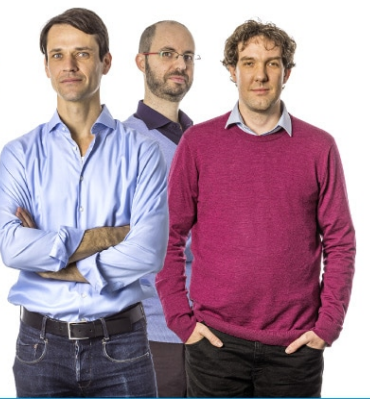




\title{
Treating linear molecules in calculations of rotation-vibration spectra
}

\author{
Cite as: J. Chem. Phys. 153, 154106 (2020); doi: 10.1063/5.0019546 \\ Submitted: 23 June 2020 - Accepted: 30 September 2020 • \\ Published Online: 16 October 2020
}

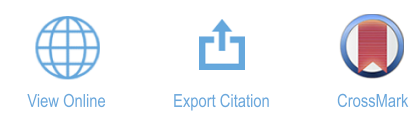

\author{
Sergei N. Yurchenko ${ }^{a)}$ (D) and Thomas M. Mellor ${ }^{\mathrm{b})}$
}

\section{AFFILIATIONS}

Department of Physics and Astronomy, University College London, London WC1E 6BT, United Kingdom

a) Electronic mail: s.yurchenko@ucl.ac.uk

b) Author to whom correspondence should be addressed: thomas.mellor.14@ucl.ac.uk

\begin{abstract}
In this article, a numerical implementation of the exact kinetic energy operator (KEO) for triatomic molecules (symmetric of $\mathrm{XY}_{2}$ type and asymmetric of YXZ-type) is presented. The implementation is based on the valence coordinates with the bisecting (XY ${ }_{2}$ type molecules) and bond-vector (YXZ) embeddings and includes the treatment of the singularity at linear geometry. The KEO is represented in a sum-of-product form. The singularity caused by the undetermined angle at the linear configuration is resolved with the help of the associated Legendre and Laguerre polynomials used as parameterized bending basis functions in the finite basis set representation. The exact KEO implementation is combined with the variational solver theoretical rovibrational energies, equipped with a general automatic symmetry-adaptation procedure and efficient basis step contraction schemes, providing a powerful computational solver of triatomic molecules for accurate computations of highly excited ro-vibrational spectra. The advantages of different basis set choices are discussed. Examples of specific applications for computing hot spectra of linear molecules are given.
\end{abstract}

Published under license by AIP Publishing. https://doi.org/10.1063/5.0019546

\section{INTRODUCTION}

TROVE (Theoretical ROVibrational Energies) is an openaccess Fortran program that uses an efficient variational approach to compute ro-vibrational energies and spectra for small polyatomic molecules. ${ }^{1}$ It is an integral part of the ExoMol toolbox ${ }^{2}$ and was used to generate hot line lists or other spectroscopic properties for 18 molecules important for atmospheric studies of exoplanets and cool stars. These applications require complete spectroscopic data at extremely high temperatures specific for atmospheres of most of the known exoplanets, typically for $1000 \mathrm{~K}-3000 \mathrm{~K}$. TROVE has been optimized for energy and intensity calculations of highly excited states, both rotationally and vibrationally. Since it can automatically generate a kinetic energy operator (KEO) for an arbitrary, semi-rigid molecule of a general structure, TROVE essentially has a black box design. Typically, the kinetic energy operator (KEO) is represented in a Taylor-type expansion around a (non-)rigid reference configuration in terms of linearized ${ }^{1}$ or curvilinear ${ }^{3}$ coordinates. Both the
KEO and the potential energy function are represented by a sumof-product form, convenient for the integrals involved in the finite basis set representation (FBR)-which it uses-where the eigenfunctions are sought as linear combinations of basis functions in a symmetrized sum-of-product form. TROVE has an automatic symmetrization tool for building symmetry-adapted basis sets. ${ }^{4}$ TROVE is also equipped with a number of auxiliary tools, including refinement of potential energy surfaces ${ }^{5}$ and thermal averaging of different properties; ${ }^{6}$ it provides efficient basis sets for quantum dynamics in the presence of an external electric field. ${ }^{7}$ Recently, TROVE has been extended to compute the electric quadrupole intensities of polyatomic molecules. ${ }^{8}$ Some disadvantages of TROVE are as follows: (i) a non-exact (expanded) representation of the KEO, (ii) a representation in rectilinear coordinates, and (iii) the automatic generation of the KEO is not capable of treating singularities appearing when the molecules become linear. The problem with the singularity could be, in principle, solved by using the $3 N-5$ approach for linear molecules, as was recently demonstrated in the case of 
$\mathrm{HCCH} .{ }^{9,10}$ However, the associated rectilinear coordinates can lead to a rather poor description of the potential energy surface and cause large errors.

Our goal is to extend TROVE to the (exact) KEO in curvilinear coordinates with adequate treatment of the singularities. To this end, here, we present an implementation of exact kinetic energy operators for triatomic, non-rigid molecules of general types $\mathrm{XY}_{2}$ and YXZ with a singularity at the linear configuration. The well-known KEOs expressed in valence coordinates in the bisecting embedding for $\mathrm{XY}_{2}$ or along one of the bonds for YXZ (see, e.g., works by Carter, Handy, and Sutcliffe, ${ }^{11}$ Littlejohn and Reinsch, ${ }^{12}$ Sutcliffe and Tennyson, ${ }^{13}$ Lukka, ${ }^{14}$ and Watson ${ }^{15}$ ) are used. To resolve the singularity in the KEO, we follow the standard approach by choosing basis functions with appropriate behavior in the vicinity of the linear geometry. This is a popular approach in the literature; see, e.g., the works of Bramley and Handy, ${ }^{16}$ Jensen, ${ }^{17}$ Tennyson et al., and Schwenke. ${ }^{19}$ Our choice here is a bending basis set built from the associated Legendre and Laguerre polynomials (see, e.g., the works of Bramley and Handy, ${ }^{16}$ Bohaček et al., ${ }^{20}$ Perić and Peyerimhoff $^{21}$ ). We show how any other appropriate basis sets can be easily constructed. In order to make this implementation compatible with the TROVE doctrine of using Taylor-like expansions in terms of 1D functions, the exact form of the triatomic's KEO is represented in a sum-of-product form. In the future, we are going to explore this form of KEO for accurate treatment of larger polyatomic molecules.

\section{KEO: AN XY,-TYPE MOLECULE}

TROVE uses an alternative to the Podolsky ${ }^{22}$ trick procedure to build and represent the KEO as developed and described in several papers; see, e.g., the works of Watson, ${ }^{15}$ Sørensen, ${ }^{23}$ and Nauts and Chapuisat. ${ }^{24}$ This approach, which we will refer to as Sørenson's, assumes a general kinetic energy operator for an $N$ atomic molecule in the form

$$
\begin{aligned}
\hat{T}= & \frac{1}{2} \sum_{\alpha=x, y, z} \sum_{\alpha^{\prime}=x, y, z} \hat{J}_{\alpha} G_{\alpha, \alpha^{\prime}}^{\mathrm{rot}}(\xi) \hat{J}_{\alpha^{\prime}} \\
& -\frac{i \hbar}{2} \sum_{\alpha=x, y, z} \sum_{n=1}^{M}\left[\hat{J}_{\alpha} G_{\alpha, n}^{\mathrm{Cor}}(\xi) \frac{\partial}{\partial \xi_{n}}+\frac{\partial}{\partial \xi_{n}} G_{\alpha, n}^{\mathrm{Cor}}(\xi) \hat{J}_{\alpha}\right] \\
& -\frac{\hbar^{2}}{2} \sum_{n=1}^{M} \sum_{n^{\prime}=1}^{M} \frac{\partial}{\partial \xi_{n}} G_{n, n^{\prime}}^{\mathrm{vib}(\xi)} \frac{\partial}{\partial \xi_{n^{\prime}}}+U(\xi)
\end{aligned}
$$

in terms of vibrational coordinates, $\boldsymbol{\xi}=\left\{\xi_{1}, \ldots, \xi_{M}\right\}$, conjugate vibrational momenta $-i \hbar \partial / \partial \xi=\left\{-i \hbar \partial / \partial \xi_{1}, \ldots,-i \hbar \partial / \partial \xi_{M}\right\}$, angular momenta operators $\hat{J}_{x}, \hat{J}_{y}, \hat{J}_{z}$, and the pseudo-potential function, $U(\xi)$, and the so-called Wilson integration volume ${ }^{25,26}$

$$
d V=\sin \theta d \xi_{1} d \xi_{2}, \ldots, d \xi_{M} d \phi d \theta d \chi
$$

with $\phi, \theta$, and $\chi$ as Euler angles. $M$ is the number of internal (vibrational) degrees of freedom, $3 N-6$ or $3 N-5$. This representation offers a number of important advantages comparing to the Euclidean normalization form. Since it assumes a symmetric, quadratic KEO in terms of the generalized momenta, the integrals of the second derivatives of the wavefunctions are not required.
Besides, this form allows for an efficient formulation of the numerical construction of the KEO for a general type of an arbitrary polyatomic molecule; see, e.g., the works of Yurchenko, Thiel, and Jensen. ${ }^{1}$ Finally, and more relevant to this work, this is the form adopted by TROVE. We, therefore, intend to represent the wellknown KEOs of triatomic molecules, as in Eq. (1). The main disadvantage of this form for our work is that the pseudo-potential function $U(\xi)$, which does not exist in the Euclidean normalization, is singular and, thus, as will be shown below, requires special treatment.

Let us consider a triatomic molecule of the type $\mathrm{XY}_{2}$ with three internal coordinates $r_{1}, r_{2}$, and $\rho$ (i.e., $\boldsymbol{\xi}=\left\{r_{1}, r_{2}, \rho\right\}$ ). Here, $r_{1}$ and $r_{2}$ are two stretching valence coordinates, and $\rho=180^{\circ}-\alpha$, with $\alpha$ being the inter-bond valence angle.

We choose the molecular frame of the $\mathrm{XY}_{2}$ molecule to be in the angle bisector embedding (bisector "gauge"), ${ }^{12}$ where the $x$ axis is selected along the bisector between the $\mathrm{X}-\mathrm{Y}_{1}$ and $\mathrm{X}-\mathrm{Y}_{2}$ vectors with the center in the nuclear center of mass and the $y$ axis orthogonal to the molecular plane for any instantaneous position of the nuclei $\mathrm{X}, \mathrm{Y}_{1}$, and $\mathrm{Y}_{2}$, as shown in Fig. 1. With this choice of the molecular frame, the $z$ axis coincides with the molecular axis $\mathrm{Y}_{1}-\mathrm{X}-\mathrm{Y}_{2}$ at the linear geometry $(\rho=0)$. The exact form of the kinetic energy operator in the bisector embedding for triatomic molecules is well known ${ }^{11,13}$ and can be represented by the following $G$ and $U$ elements:

1. The vibrational part

$$
\begin{aligned}
G_{1,1}^{\mathrm{vib}} & =G_{2,2}^{\mathrm{vib}}=\frac{1}{\mu_{X Y}}, \\
G_{1,2}^{\mathrm{vib}} & =G_{2,1}^{\mathrm{vib}}=-\frac{\cos \rho}{m_{X}}, \\
G_{1,3}^{\mathrm{vib}} & =G_{3,1}^{\mathrm{vib}}=\frac{\sin \rho}{r_{2} m_{X}}, \\
G_{2,3}^{\mathrm{vib}} & =G_{3,2}^{\mathrm{vib}}=\frac{\sin \rho}{r_{1} m_{X}}, \\
G_{3,3}^{\mathrm{vib}} & =\frac{1}{\mu_{X Y}}\left(\frac{1}{r_{1}^{2}}+\frac{1}{r_{2}^{2}}\right)+\frac{2 \cos \rho}{r_{1} r_{2} m_{X}} .
\end{aligned}
$$

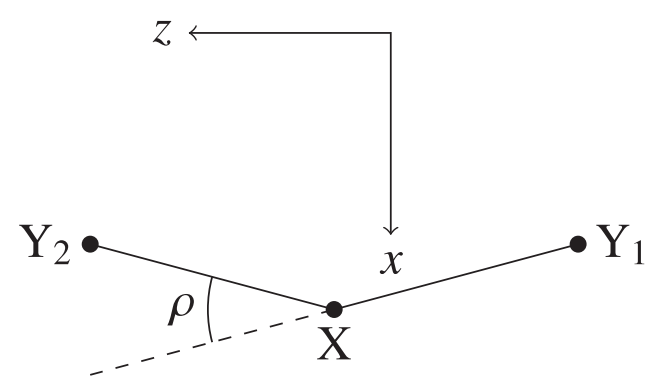

FIG. 1. The molecular axis orientation for the symmetric molecule in the bisecting frame, where the $x$ axis is parallel to the bisector of the angle $\angle Y_{1}-X-Y_{2}$. For clarity, the position of the axes is not at the center of mass of the molecule. 
2. The pseudo-potential function

$$
U\left(r_{1}, r_{2}, \rho\right)=-\frac{\hbar^{2}}{8 \sin ^{2}(\rho)}\left\{\frac{1+\sin ^{2}(\rho)}{\mu_{X Y}}\left[\frac{1}{r_{1}^{2}}+\frac{1}{r_{2}^{2}}\right]+\frac{2 \cos ^{3}(\rho)}{r_{1} r_{2} m_{X}}\right\} .
$$

3. The Coriolis part (only non-zero elements):

$$
\begin{aligned}
G_{1, y}^{\text {Cor }} & =-\frac{\sin \rho}{2 r_{2} m_{X}}, \\
G_{2, y}^{\text {Cor }} & =\frac{\sin \rho}{2 r_{1} m_{X}}, \\
G_{3, y}^{\text {Cor }} & =\frac{1}{2 \mu_{X Y}}\left(\frac{1}{r_{1}^{2}}-\frac{1}{r_{2}^{2}}\right) .
\end{aligned}
$$

4. The rotational part (only non-zero elements):

$$
\begin{aligned}
G_{x, x}^{\mathrm{rot}} & =\frac{1}{4 \cos ^{2}(\rho / 2)}\left[\frac{1}{\mu_{X Y}}\left(\frac{1}{r_{1}^{2}}+\frac{1}{r_{2}^{2}}\right)-2 \frac{1}{m_{X} r_{1} r_{2}}\right], \\
G_{x, z}^{\mathrm{rot}} & =G_{z, x}^{\mathrm{rot}}=\frac{1}{2 \mu_{X Y} \sin \rho}\left(\frac{1}{r_{2}^{2}}-\frac{1}{r_{1}^{2}}\right), \\
G_{y, y}^{\mathrm{rot}} & =\frac{1}{4}\left[\frac{1}{\mu_{X Y}}\left(\frac{1}{r_{1}^{2}}+\frac{1}{r_{2}^{2}}\right)-2 \frac{\cos \rho}{m_{X} r_{1} r_{2}}\right], \\
G_{z, z}^{\mathrm{rot}} & =\frac{\cos ^{2}(\rho / 2)}{\sin ^{2} \rho}\left[\frac{1}{\mu_{X Y}}\left(\frac{1}{r_{1}^{2}}+\frac{1}{r_{2}^{2}}\right)+\frac{2}{m_{X} r_{1} r_{2}}\right] .
\end{aligned}
$$

In Eqs. (2)-(14), $\mu_{X Y}$ is the reduced mass, given by

$$
\frac{1}{\mu_{X Y}}=\frac{1}{m_{X}}+\frac{1}{m_{Y}},
$$

and we numbered the three coordinates $r_{1}, r_{2}$, and $\rho$ as 1,2 , and 3, respectively.

As mentioned above, the Wilson integration volume was used and is the natural volume element for the Sørensen method. It also ensures that the KEO is in the form of Eq. (1) and is thus compatible with the preexisting formulation in TROVE. If one, instead, were to use the Euclidean integration volume, given by

$$
d V^{\prime}=r_{1}^{2} r_{2}^{2} \sin \rho \sin \theta d r_{1} d r_{2} d \phi d \theta d \chi
$$

the expression of the KEO $\hat{T}_{E}$ would be given by Eqs. (14)-(18) of Tennyson and Sutcliffe. ${ }^{27}$ To transform into the Wilson representation, one changes $\hat{T}_{E}$ according to

$$
\hat{T}=\left(r_{1}^{2} r_{2}^{2} \sin \rho\right)^{1 / 2} \hat{T}_{E}\left(r_{1}^{2} r_{2}^{2} \sin \rho\right)^{-1 / 2},
$$

which leads to Eqs. (1)-(14), and one also changes the basis functions from $\phi\left(r_{1}, r_{2}, \rho\right)$ to $r_{1} r_{2} \sqrt{\sin \rho} \phi\left(r_{1}, r_{2}, \rho\right)$. Thus, it becomes necessary to include a $\sqrt{\sin \rho}$ term in the basis functions to compensate for the absence of $\sin \rho$ in the volume element; this resolves the apparent singularities, as described below.

\section{A. Singularity}

We will assume that the solution of the eigenvalue problem for the Hamiltonian

$$
\hat{H}=\hat{T}+V(\xi)
$$

for an interatomic potential energy function $V(\xi)$ will be found variationally, i.e., by constructing and diagonalizing a Hamiltonian matrix $\mathbf{H}$ on an appropriate basis set. When evaluating the integrals of the kinetic energy part $\hat{T}$ involving vibrational momentum operators $\hat{p}_{i}=-i \hbar \partial / \partial \xi_{i}$ in Eq. (1), we will change the direction of $\hat{p}_{i}$ to the left by applying integration by parts, ${ }^{28}$

$$
\begin{aligned}
-i \hbar \int_{a}^{b} \phi\left(\xi_{i}\right) \frac{\partial}{\partial \xi_{i}} G_{i, \lambda}(\xi) \hat{\Pi}_{\lambda} \phi^{\prime}\left(\xi_{i}\right) d \xi_{i} \\
=-\left.i \hbar \phi\left(\xi_{i}\right) G_{i, \lambda}(\xi) \hat{\Pi}_{\lambda} \phi^{\prime}\left(\xi_{i}\right)\right|_{a} ^{b} \\
\quad+i \hbar \int_{a}^{b} \frac{\partial \phi\left(\xi_{i}\right)}{\partial \xi_{i}} G_{i, \lambda}(\xi) \hat{\Pi}_{\lambda} \phi^{\prime}\left(\xi_{i}\right) d \xi_{i} \\
=i \hbar \int_{a}^{b} \frac{\partial \phi\left(\xi_{i}\right)}{\partial \xi_{i}} G_{i, \lambda}(\xi) \hat{\Pi}_{\lambda} \phi^{\prime}\left(\xi_{i}\right) d \xi_{i} \\
=-\int_{a}^{b} \phi\left(\xi_{i}\right) \hat{p}_{i}^{\leftarrow} G_{i, \lambda}(\xi) \hat{\Pi}_{\lambda} \phi^{\prime}\left(\xi_{i}\right) d \xi_{i},
\end{aligned}
$$

where we assumed that the first part vanishes due to the boundary conditions for the bound solution and used the notation

$$
\phi\left(\xi_{i}\right) \hat{p}_{i}^{\leftarrow}=-i \hbar \frac{\partial \phi\left(\xi_{i}\right)}{\partial \xi_{i}} .
$$

Here, $\hat{\Pi}_{\lambda}$ is a generalized momentum operator, $\hat{\boldsymbol{\Pi}}=\left\{\hat{p}_{1}, \hat{p}_{2}, \hat{p}_{3}, \hat{J}_{x}\right.$, $\left.\hat{J}_{y}, \hat{J}_{z}\right\}$. The pseudo-potential matrix elements are, of course, in the form

$$
\int_{a}^{b} \phi\left(\xi_{i}\right) U(\xi) \phi^{\prime}\left(\xi_{i}\right) d \xi_{i}
$$

The singular terms in the KEO above are $G_{x, x}^{\text {rot }}, G_{z, z}^{\text {rot }}, G_{x, z}^{\text {rot }}$, and $U$ from Eqs. (11), (14), (12), and (7), respectively. Both the pure vibrational pseudo-potential function $U$ and the rotational KE factor $G_{z, z}^{\text {rot }}$ have the singularity of the type $\frac{1}{\sin ^{2} \rho}\left(\sim 1 / \rho^{2}\right)$, while the singularity of $G_{x, z}^{\mathrm{rot}}\left(r_{1}, r_{2}, \rho\right)$ is of the type $\frac{1}{\sin \rho}(\sim 1 / \rho)$. The singularity of $G_{x, x}^{\text {rot }}\left(r_{1}, r_{2}, \rho\right)$ is of the type $\frac{1}{\cos ^{2}(\rho / 2)}$, which is singular at $\rho=180^{\circ}$.

\section{B. Associated Legendre polynomials}

The traditional, $3 \mathrm{~N}-6$-type approach to resolve the singularity in the KEO is done by properly selecting the basis set functions that make the corresponding matrix elements of the KEO finite. Among the most popular choices are the associated Legendre $P_{n}^{k}(x)$ and the associated Laguerre $L_{n}^{l}(x)$ polynomials. The orthogonal basis functions constructed from the real normalized associated Legendre polynomials have the form

$$
\psi_{n}^{(k)}(\rho)=\sqrt{\sin \rho} \bar{P}_{n}^{(k)}(\cos \rho),
$$

with the normalization condition 
$\int_{-1}^{1}\left[\bar{P}_{n}^{(k)}(x)\right]^{2} d x=\int_{0}^{\pi}\left[\bar{P}_{n}^{(k)}(\cos \rho)\right]^{2} \sin \rho d \rho=\int_{0}^{\pi}\left|\psi_{n}^{(k)}\right|^{2} d \rho=1$

where $x=\cos \rho$. Here, the rotational-angular basis $(J>0)$ is then given by

$$
\psi_{n, J, k, m}^{\mathrm{rv}}=\psi_{n}^{(k)}|J, k, m\rangle,
$$

where $k$ is both the rotational quantum number and the Legendre index.

We use the factor $\sqrt{\sin \rho}$ in Eq. (17) to resolve the apparent singularities by rewriting the Hamiltonian matrix elements so that the integrals are in the form of Eqs. (15) and (16) with a modified $\boldsymbol{G}$ matrix and pseudo-potential function $U$ (vibrational part of the KEO) where $\bar{P}_{n}^{(k)}(\cos \rho)$ takes the place of $\phi\left(\xi_{i}\right)$. We thus obtain

$$
\begin{aligned}
& \frac{\hbar^{2}}{2} \frac{\partial \psi_{n}^{(k)}}{\partial \rho} G_{3,3} \frac{\partial \psi_{m}^{(k)}}{\partial \rho}+\psi_{n}^{(k)} U \psi_{m}^{(k)} \\
& =\frac{\hbar^{2}}{2} \sin \rho \frac{\partial \bar{P}_{n}^{(k)}}{\partial \rho} G_{3,3} \frac{\partial \bar{P}_{m}^{(k)}}{\partial \rho}+\bar{P}_{n}^{(k)} \tilde{U} \bar{P}_{m}^{(k)}
\end{aligned}
$$

where a new pseudo-potential term for the basis $\bar{P}_{n}^{(k)}$ is given by

$$
\begin{aligned}
\tilde{U} & =\frac{\hbar^{2}}{8}\left[-2 \cos \rho \frac{\partial G_{3,3}}{\partial \rho}+\left(2 \sin \rho+\frac{\cos ^{2} \rho}{\sin \rho}\right) G_{3,3}\right]+U \sin \rho \\
& =\hbar^{2} \frac{\sin (2 \rho)}{2 r_{1} r_{2} m_{X}}
\end{aligned}
$$

which has a very compact form and is not singular.

The singularity of the term $G_{z, z} \hat{J}_{z}^{2}$ in Eq. (14) is not relevant for $k=0$ due to the zero action of $\hat{J}_{z}$ on the rotational rigid rotor basis functions $|J, k, m\rangle$. The associated Legendre polynomials $\bar{P}_{n}^{(k)}(k>0)$ have the following form: ${ }^{16}$

$$
\bar{P}_{n}^{(k)}(\cos \rho)=\sin ^{k}(\rho) \tilde{P}_{n}^{(k)}(\cos \rho),
$$

and therefore, the singularity in Eq. (17) is automatically resolved by the factor $\sin ^{k}(\rho)$ in the definition of $\bar{P}_{n}^{k}$ (see, e.g., the work of Sutcliffe and Tennyson ${ }^{13}$ ), which cancels the term $1 / \sin ^{2}(\rho)$ in Eq. (14) for any $k \geq 1$. Moreover, the combination

$$
-\frac{\hbar^{2}}{2} G_{3,3} \frac{\partial^{2} \psi_{m}^{(k)}}{\partial \rho^{2}}+\frac{\hbar^{2}}{2} k^{2} G_{z, z} \psi_{n}^{(k)}
$$

is non-singular for $\psi_{m}^{(k)}$ from Eq. (17), which has been explored in the literature (see, e.g., the work of Jensen ${ }^{29}$ ). To show this, first note that

$$
\frac{\partial \bar{P}_{n}^{(k)}(\cos \rho)}{\partial \rho}=k \frac{\cos \rho}{\sin \rho} \bar{P}_{n}^{(k)}+\bar{P}_{n}^{(k+1)} .
$$

The terms containing the $k^{2}$ factor from Eq. (21)

$$
-\frac{\hbar^{2}}{2} G_{3,3} \frac{\cos ^{2} \rho}{\sin ^{2} \rho} k^{2}
$$

cancel the divergent term of $k^{2} G_{z, z}$ at $\rho \rightarrow 0$.
In order to derive the matrix elements in a non-singular form for the basis set in Eq. (17), let us factorize out the $\sin \rho$ term from $\psi_{n}^{(k)}(\rho)$ and $\tilde{P}_{n}^{(k)}(\rho)$ by introducing the following notation:

$$
\chi_{n}^{(k)}(\rho)=\sin ^{k-1}(\rho) \tilde{P}_{n}^{(k)}(\cos \rho) \quad(k>0),
$$

which is related to $\psi_{n}^{(k)}$ as

$$
\psi_{n}^{(k)}(\rho)=\sqrt{\sin \rho} \bar{P}_{n}^{(k)}(\cos \rho)=\sqrt{\sin \rho} \sin \rho \chi_{n}^{(k)}(\rho) .
$$

Using this notation, Eq. (18) becomes

$$
\begin{gathered}
\frac{\hbar^{2}}{2} \frac{\partial \psi_{n}^{(k)}}{\partial \rho} G_{3,3} \frac{\partial \psi_{n^{\prime}}^{(k)}}{\partial \rho}+\psi_{n}^{(k)} U \psi_{n^{\prime}}^{(k)}+\frac{\hbar^{2}}{2} G_{z, z} k^{2} \psi_{n}^{(k)} \psi_{n^{\prime}}^{(k)} \\
=\frac{\hbar^{2}}{2} \sin \rho \frac{\partial \bar{P}_{n}^{(k)}}{\partial \rho} G_{3,3} \frac{\partial \bar{P}_{n^{\prime}}^{(k)}}{\partial \rho}+\bar{P}_{n}^{(k)} \tilde{U} \bar{P}_{n^{\prime}}^{(k)} \\
+\frac{\hbar^{2}}{2} \tilde{G}_{z, z} k^{2} \chi_{n}^{(k)} \chi_{n^{\prime}}^{(k)} \sin \rho,
\end{gathered}
$$

where both $\tilde{U}$, given by Eq. (19), and

$$
\tilde{G}_{z, z}=G_{z, z} \sin ^{2} \rho=\cos ^{2}(\rho / 2)\left[\frac{1}{\mu_{X Y}}\left(\frac{1}{r_{1}^{2}}+\frac{1}{r_{2}^{2}}\right)+\frac{2}{m_{X} r_{1} r_{2}}\right]
$$

are non-singular.

For evaluation of matrix elements of other KEO terms containing $\hat{p}_{3}$, we define the first derivative of $\psi_{n}^{(k)}$ with respect to $\rho$ in the following non-singular form:

$$
d_{n}^{(k)}(\rho) \equiv \sqrt{\sin \rho} \frac{\partial \psi_{n}^{(k)}}{\partial \rho}=\frac{1}{2} \cos \rho \bar{P}_{n}^{(k)}+\sin \rho \frac{\partial \bar{P}_{n}^{(k)}}{\partial \rho} .
$$

Here, we assumed that $\sqrt{\sin \rho}$ can always be borrowed from the counterpart bra or ket vector in Eq. (23). Second derivatives of the wavefunctions are not required due to Sørenson's form of the $\mathrm{KEO}$ in Eq. (1) and the change in the direction of $\hat{p}_{i}$ according to Eq. (15).

The matrix elements of all the factors of the Hamiltonian in the associated Legendre basis of $\psi_{n}^{(k)}$ are then given by

$$
\int_{0}^{\pi} \psi_{n}^{(k)} V \psi_{n^{\prime}}^{(k)} d \rho
$$

$$
\int_{0}^{\pi}\left[\sin \rho \frac{\partial \bar{P}_{n}^{(k)}}{\partial \rho} G_{3,3} \frac{\partial \bar{P}_{n^{\prime}}^{(k)}}{\partial \rho}+\frac{2}{\hbar^{2}} \bar{P}_{n}^{(k)} \tilde{U} \bar{P}_{n^{\prime}}^{(k)}+k^{2} \sin \rho \chi_{n}^{(k)} \tilde{G}_{z, z} \chi_{n^{\prime}}^{(k)}\right] d \rho
$$

$$
\begin{gathered}
\int_{0}^{\pi} \psi_{n}^{(k)} G_{i, 3} \frac{\partial \psi_{n^{\prime}}^{(k)}}{\partial \rho} d \rho=\int_{0}^{\pi} \bar{P}_{n}^{(k)} G_{i, 3} d_{n^{\prime}}^{(k)} d \rho \quad(i=1,2), \\
\int_{0}^{\pi} \psi_{n}^{(k)} \frac{\partial}{\partial \rho} G_{3, i} \psi_{n^{\prime}}^{(k)} d \rho=-\int_{0}^{\pi} d_{n}^{(k)} G_{3, i} \bar{P}_{n^{\prime}}^{(k)} d \rho \quad(i=1,2),
\end{gathered}
$$




$$
\begin{gathered}
\int_{0}^{\pi} \psi_{n}^{(k)} G_{i, j} \psi_{n^{\prime}}^{(k)} d \rho \quad(i, j=1,2) \\
\int_{0}^{\pi} \bar{P}_{n}^{(k)} \tilde{G}_{x, z} \bar{P}_{n^{\prime}}^{\left(k^{\prime}\right)} d \rho, \quad \int_{0}^{\pi} \bar{P}_{n}^{(k)} \tilde{G}_{z, x} \bar{P}_{n^{\prime}}^{\left(k^{\prime}\right)} d \rho \quad\left(\left|k-k^{\prime}\right|=1\right) \\
\int_{0}^{\pi} \psi_{n}^{(k)} G_{x, x} \psi_{n^{\prime}}^{\left(k^{\prime}\right)} d \rho, \quad \int_{0}^{\pi} \psi_{n}^{(k)} G_{y, y} \psi_{n^{\prime}}^{\left(k^{\prime}\right)} d \rho \quad\left(\left|k-k^{\prime}\right|=0,2\right) \\
\int_{0}^{\pi} \psi_{n}^{(k)} \tilde{G}_{z, z} \psi_{n^{\prime}}^{\left(k^{\prime}\right)} d \rho \quad\left(\left|k-k^{\prime}\right|=0\right) \\
\left.=\int_{0}^{\pi} G_{3, y}^{(k)} \frac{\partial}{\partial \rho} G_{3, y} \psi_{n^{\prime}}^{\left(k^{\prime}\right)}+\psi_{n}^{(k)} G_{y, 3}^{(k)} \frac{\partial}{\partial \rho} \psi_{n^{\prime}}^{\left(k^{\prime}\right)}-d_{n}^{(k)} \bar{P}_{n^{\prime}}^{\left(k^{\prime}\right)}\right) d \rho \quad\left(\left|k-k^{\prime}\right|=1\right) \\
\int_{0}^{\pi} \psi_{n}^{(k)} G_{y, i} \psi_{n^{\prime}}^{\left(k^{\prime}\right)} d \rho, \quad \int_{0}^{\pi} \psi_{n}^{(k)} G_{i, y} \psi_{n^{\prime}}^{\left(k^{\prime}\right)} d \rho \quad\left(i=1,2,\left|k-k^{\prime}\right|=1\right)
\end{gathered}
$$

where

$$
\tilde{G}_{z, z}=\sin ^{2} \rho G_{z, z}, \quad \tilde{G}_{x, z}=\sin \rho G_{x, z}, \quad \tilde{G}_{z, x}=\sin \rho G_{z, x} .
$$

In Eq. (34), the property $G_{3, y}=G_{y, 3}$ was used.

It should be noted that the $G_{x, x}$ term in Eq. (11) has a singularity that leads to a divergence at $\rho=180^{\circ}$ (the $\mathrm{XY}_{2}$ molecule is fully bent, $\alpha=0$ ), which cannot be resolved for the associated Legendre polynomials when $k=0$ and $J>0$. In practice, however, this divergence is not important because this geometry corresponds to a very high energy. ${ }^{13}$ For example, before entering the divergent integrals of $G_{x, x}$ in Eq. (32), the primitive basis functions $\sqrt{\sin \rho} \bar{P}_{n}^{(k)}(\cos \rho)$ can be optimized by eigen-solving the pure angular Schrödinger equation for a realistic angular potential energy function $\bar{V}(\rho)$, as will be shown below. The optimized solution usually vanishes at the limit $\rho \rightarrow 180^{\circ}$ faster than $1 / \cos ^{2}(\rho / 2)$ in Eq. (11).

\section{Basis set generation}

The typical way to generate the associated Legendre polynomials is by recurrence relations. ${ }^{30,31}$ However, having in mind a generalization to arbitrary polynomials (see below) and also to represent them in the form $\bar{P}_{n}^{(k)}(\cos \rho)$ as in Eq. (20) required for the singularity canceling described above, we generate $\bar{P}_{n}^{(k)}(\cos \rho)$ from a non-orthonormal basis set of the different powers of $\cos ^{n} \rho$ using the Gram-Schmidt orthogonalization procedure in conjunction with the ortho-normality condition,

$$
\int_{0}^{\pi} \sin ^{2 k+1} \rho \tilde{P}_{n}^{(k)}(\cos \rho) \tilde{P}_{n^{\prime}}^{(k)}(\cos \rho) d \rho=\delta_{n, n^{\prime}} .
$$

For any value of $k\left(k=0, \ldots, k_{\max }\right)$, we start from a normalized basis function $\cos ^{n} \rho$ with the weight factor $\sin ^{2 k+1}(\rho)$,

$$
F_{n}^{(k)}(\cos \rho)=\frac{\cos ^{n} \rho}{\sqrt{\int_{0}^{\pi} \sin ^{2 k+1} \rho \cos ^{2 n} \rho d \rho}},
$$

and then follow the Gram-Schmidt process to generate $\tilde{P}_{n}^{(k)}(\cos \rho)$ $\left(n=0, \ldots, n_{\max }\right)$ recursively as given by

$$
\begin{aligned}
\tilde{P}_{n}^{(k)}(\cos \rho)= & F_{n}^{(k)}(\cos \rho)-\sum_{m=0}^{n-1} \int_{0}^{\pi} \sin ^{2 k+1} \rho F_{n}^{(k)} \\
& \times \tilde{P}_{m}^{(k)}(\cos \rho) d \rho .
\end{aligned}
$$

The numerical construction of the basis set by the Gram-Schmidt orthogonalization provides a flexible and robust way to generate orthonormal basis sets on the fly, as was used by Schiffel and Manthe $^{32}$ to generate the cot-DVR (discrete variable representation) basis set.

\section{Associated Laguerre polynomials}

The disadvantage of the Legendre-based basis functions is that they are not sufficiently compact for representing the bending motion. A more physically motivated basis set that also allows for the full resolution of the $\rho=0$ singularity in Eqs. (7), (12), and (14) is the one based on the associated Laguerre polynomials $L_{n}^{(l)}(\rho)$ (see, e.g., the work of Perić and Peyerimhoff ${ }^{21}$ ).

Laguerre bending basis functions constructed from the (real) associated Laguerre polynomials $L_{n}^{(l)}(\rho)$ are given by

$$
\psi_{n}^{(l)}(\rho)=C_{n, l} \rho^{l+1 / 2} L_{n}^{(l)}\left(a \rho^{2}\right) e^{-a \rho^{2} / 2}
$$

and are normalized as

$$
\int_{0}^{\infty} \psi_{n}^{(l)}(\rho)^{2} d \rho=1
$$

with

$$
C_{n, l}=\sqrt{\frac{2 n !}{(n+l) !}} a^{\frac{l+1}{2}},
$$

where $a$ is a structural parameter. Due to the bending nature of $\rho$ and also the singularity at $\rho=\pi$, we will have to restrict the integration range in Eq. (40) to $\rho=\left[0, \ldots, \rho_{\max }\right]$, where $\rho_{\max }<180^{\circ}$ with $C_{n, l}$ obtained via numerical normalization of $\psi_{n}^{(l)}(\rho)$.

Following the example of the Legendre basis set, we now show how the Laguerre basis functions resolve the singularity in the $\mathrm{KE}$ terms $G_{z, z}^{\text {rot }}, G_{x, z}^{\text {rot }}$, and $U$. In this case, $\sqrt{\rho}$ takes the place of $\sqrt{\sin \rho}$. The change in the basis function that results from using the Wilson integration volume is thus absorbed into the basis function implicitly. As mentioned above, these three KE components are combined with the Laguerre basis functions $\psi_{n}^{(l)}$ as given by

$$
\begin{aligned}
\frac{\hbar^{2}}{2} \frac{\partial \psi_{n}^{(l)}}{\partial \rho} G_{3,3} \frac{\partial \psi_{n^{\prime}}^{(l)}}{\partial \rho}+\psi_{n}^{(l)} U \psi_{n^{\prime}}^{(l)}+\frac{\hbar^{2}}{2} G_{z, z} k^{2} \psi_{n}^{(l)} \psi_{n^{\prime}}^{(l)} \\
=\frac{\hbar^{2}}{2} \rho \frac{\partial \phi_{n}^{(l)}}{\partial \rho} G_{3,3} \frac{\partial \phi_{n^{\prime}}^{(l)}}{\partial \rho}+\phi_{n}^{(l)} \tilde{U} \phi_{n^{\prime}}^{(l)} \\
+\frac{\hbar^{2}}{2} \rho \tilde{G}_{z, z} k^{2} \chi_{n}^{(l)} \chi_{n^{\prime}}^{(l)},
\end{aligned}
$$

where the substitution 


$$
\hat{J}_{z}^{2} G_{z, z}|J, k, m\rangle=\hbar^{2} k^{2} G_{z, z}|J, k, m\rangle
$$

was used in conjunction with the rotational basis function $|J, k, m\rangle$ and the following functions were introduced,

$$
\begin{gathered}
\phi_{n}^{(l)}(\rho)=C_{n, l} \rho^{l} L_{n}^{(l)}\left(a \rho^{2}\right) e^{-a \rho^{2} / 2} \quad(l \geq 0), \\
\chi_{n}^{(l)}(\rho)=C_{n, l} \rho^{l-1} L_{n}^{(l)}\left(a \rho^{2}\right) e^{-a \rho^{2} / 2} \quad(l>0),
\end{gathered}
$$

which are related to $\psi_{n}^{(l)}$ as

$$
\psi_{n}^{(l)}(\rho)=\sqrt{\rho} \phi_{n}^{(l)}(\rho)=\sqrt{\rho} \rho \chi_{n}^{(l)}(\rho) .
$$

The exponential term in Eq. (39) ensured that the basis function vanishes at $\rho_{\max }$ so that, when integrating by parts, the boundary term at $\rho_{\max }$ in Eq. (15) can be ignored. Analogously to the treatment using the Legendre polynomials, we have also introduced the rotational KE factor $\tilde{G}_{z, z}$

$$
\tilde{G}_{z, z}=G_{z, z} \rho^{2}
$$

and a new pseudo-potential term

$$
\tilde{U}=U \rho+\frac{\hbar^{2}}{8}\left[-2 \frac{\partial G_{3,3}}{\partial \rho}+\frac{G_{3,3}}{\rho}\right] .
$$

It is easy to see from Eqs. (7) and (14) that neither $\tilde{G}_{z, z}$ nor $\tilde{U}$ is singular,

$$
\begin{aligned}
\tilde{G}_{z, z} & \approx \frac{1}{\mu_{X Y}}\left[\frac{1}{r_{1}^{2}}+\frac{1}{r_{2}^{2}}\right]+\frac{2}{r_{1} r_{2} m_{X}}+O\left(\rho^{2}\right), \\
\tilde{U} & \approx \hbar^{2}\left[-\frac{1}{6 \mu_{X Y}}\left(\frac{1}{r_{1}}+\frac{1}{r_{2}}\right)+\frac{2}{3 r_{1} r_{2} m_{X}}\right] \rho+O\left(\rho^{3}\right) .
\end{aligned}
$$

One can see from Eqs. (43) and (44) that the term $\tilde{G}_{z, z} k^{2} \phi_{n}^{(l)} \phi_{n^{\prime}}^{(l)}$ in Eq. (41) is never singular either for non-zero values of $k$ if we assume that $l=|k|$.

The matrix elements of all the factors of the Hamiltonian in the basis of $\psi_{n}^{(l)}$ are then given by Eqs. (26)-(35) with $d_{n}^{(l)}(\rho)$ given analogously to Eq. (25) by

$$
d_{n}^{(l)}(\rho) \equiv \sqrt{\rho} \frac{\partial \psi_{n}^{(k)}}{\partial \rho}=\frac{1}{2} \phi_{n}^{(l)}+\rho \frac{\partial \phi_{n}^{(l)}}{\partial \rho}
$$

and $\sin \rho$ in Eq. (27) replaced by $\rho$.

Our next step is to optimize the Laguerre basis functions by solving a $1 \mathrm{D}$ bending Schrödinger equation for the model Hamiltonian operator

$$
\hat{H}^{1 \mathrm{D}}(\rho)=-\frac{\hbar^{2}}{2} \frac{\partial}{\partial \rho} \bar{G}_{3,3}(\rho) \frac{\partial}{\partial \rho}+\frac{\hbar^{2}}{2} \bar{G}_{z, z}(\rho) k^{2}+\bar{U}(\rho)+\bar{V}(\rho)
$$

variationally on the basis $\psi_{n}^{(l)}(\rho)$. Here, $\bar{G}_{3,3}(\rho), \bar{G}_{z, z}(\rho), \bar{U}$, and $\bar{V}$ are obtained from the corresponding 3D forms by setting $r_{1}=r_{2}$ $=r_{\mathrm{e}}\left(r_{\mathrm{e}}\right.$ is the equilibrium bond length). The structural parameter $a$ is chosen as

$$
a=\sqrt{\frac{f_{2}}{g_{0}}}
$$

where

$$
f_{2}=\left.\frac{1}{2} \frac{\partial^{2} \bar{V}(\rho)}{\partial \rho^{2}}\right|_{\rho=0}
$$

and $g_{0}=\bar{G}_{3,3}(\rho=0)$ is the equilibrium value. With this choice of $a$, $\psi_{n}^{(l)}(\rho)$ in Eq. (39) are eigenfunctions of the model 1D Hamiltonian operator,

$$
\hat{H}^{\text {model }}=-\frac{\hbar^{2}}{2} g_{0} \frac{\partial^{2}}{\partial \rho^{2}}+\frac{\hbar^{2}}{8} g_{0} \frac{\left(4 l^{2}-1\right)}{\rho^{2}}+f_{2} \rho^{2} .
$$

This 1D Hamiltonian operator is equivalent to that of the 2D isotropic harmonic oscillator.

We solve the Schrödinger equation for the Hamiltonian operator $\hat{H}^{1 \mathrm{D}}$ in Eq. (47) variationally on the basis of $\psi_{n}^{(l)}(\rho)$ with $l \geq 0$. The matrix elements of $\hat{H}^{1 \mathrm{D}}$ are computed numerically on a grid of $\rho=0, \ldots, \rho_{\max }$ in quadruple precision. The associated Laguerre polynomial is generated using a Fortran subroutine from the library by John Burkardt. ${ }^{33}$ The Hamiltonian matrix is diagonalized with the quadruple precision. The eigenfunctions $\Phi_{n}^{(l)}$ of $\hat{H}^{1 \mathrm{D}}$ are then used as optimized basis functions for the solution of the full ro-vibrational problem (see Sec. IV).

Figure 2 shows selected Legendre $\psi_{n}^{(k=0)}(\rho)$ from Eq. (17) and Laguerre $\psi_{n}^{(l=0)}(\rho)$ basis functions from Eq. (39) for $n=10$ and compares them to the corresponding optimized eigenfunctions $\Phi_{12}^{(0)}$ for $\mathrm{CO}_{2}$ as an example. Here, a relatively large primitive basis function (associated Laguerre or Legendre polynomials) of $n_{\max }=48$ was used. The Laguerre basis function $\psi_{n}^{(l=0)}$ has a much more compact form than the Legendre basis function and is very close to the optimized solution illustrating the better quality of the Laguerre basis set. Even the well-optimized Legendrebased eigenfunction $\Phi_{n}^{(0)}$ (Legendre) shows clear oscillations in the region of small inter-bond angle, which should be forbidden due to the high potential energy. This artifact of the Legendre basis is not eliminated completely even with the relatively large set. The Laguerre-based eigensolution gives a clean eigensolution and, therefore, should be more preferable for high vibrational excitations. Numerical noise in calculations of matrix elements of dipole moments is known to lead to intensity instabilities in overtone spectra of diatomics ${ }^{34}$ and have been recently investigated in the case of triatomics. $^{33}$

Figure 3 illustrates the importance of the centrifugal term $\frac{\hbar^{2}}{2} \bar{G}_{z, z}(\rho) k^{2}$ when optimizing the bending basis set for $k>0$. A more detailed discussion of the calculations for $\mathrm{CO}_{2}$ is given below.

\section{E. Factorization of the KEO}

The KEO factors in Eqs. (2)-(6), Eq. (7), Eqs. (8)-(10), and Eqs. (11)-(14) have a sum-of-product form, which is convenient for integrations on 1D basis sets. We can also take advantage of the non-rigid reference approach by Hougen, Bunker, and Johns ${ }^{36}$ 

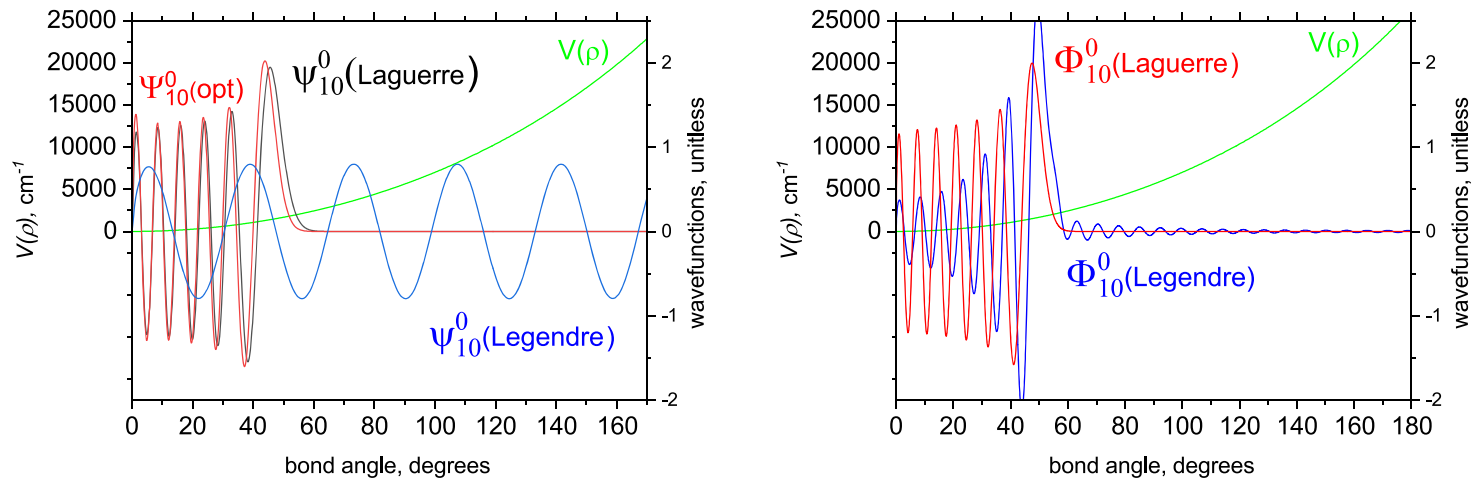

FIG. 2. Left display: Legendre polynomial based basis function $\phi_{10}^{(0)}$ and Laguerre-polynomial-based basis functions $\psi_{10}^{(0)}$ for $\mathrm{CO}_{2}$. Right display: The corresponding optimized wavefunctions $\Phi_{20}^{(0)}$ (Legendre) and $\Phi_{20}^{(0)}$ (Laguerre). The optimized Legendre-based wavefunctions show oscillations in the forbidden (high energy) region. The 1D potential energy function $V(\rho)$ is shown as reference. The computational details are defined in Sec. V.

implemented in TROVE by representing the KEO as a formal expansion around the non-rigid reference configuration defined by $\rho$ with respect to the variables $1 / r_{i}$ and $1 / r_{i}^{2}$,

$$
G_{\lambda, \lambda^{\prime}}\left(r_{1}, r_{2}, \rho\right)=\sum_{k, m=0,1,2} G_{k, m}^{\left(\lambda, \lambda^{\prime}\right)}(\rho) f_{k}\left(r_{1}\right) f_{m}\left(r_{2}\right)
$$

where

$$
\begin{aligned}
& f_{1}(r)=1, \\
& f_{2}(r)=1 / r \\
& f_{3}(r)=1 / r^{2} .
\end{aligned}
$$
given by

The vibrational expansion factors $G_{k, m}^{\lambda, \lambda^{\prime}}\left(\lambda, \lambda^{\prime}=1,2,3\right)$ are then

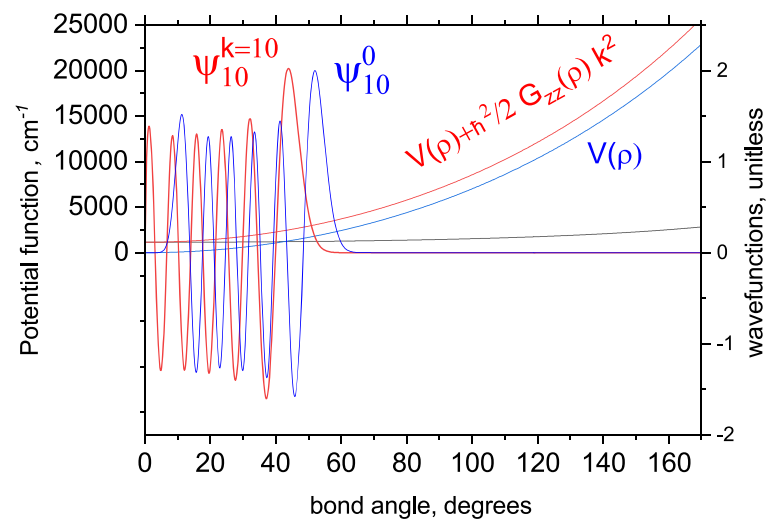

FIG. 3. The effective potential energy function with the centrifugal term for $k=10$ included, $\Phi_{10}^{(10)}$ (optimized) basis functions for $\mathrm{CO}_{2}$, compared to the corresponding potential energy function $V(\rho)$ and $\Phi_{10}^{(0)}$ for $k=0$. The centrifugal term is shown as a near flat black line.

$$
\begin{aligned}
& G_{0,0}^{1,1}=G_{0,0}^{2,2}=G_{2,0}^{3,3}=G_{0,2}^{3,3}=\frac{\hbar^{2}}{\mu_{X Y}}, \\
& G_{0,0}^{1,2}=G_{0,0}^{2,1}=-\cos \rho \frac{\hbar^{2}}{m_{X}}, \\
& G_{1,0}^{1,3}=G_{0,1}^{2,3}=G_{1,0}^{3,1}=G_{0,1}^{3,2}=\sin \rho \frac{\hbar^{2}}{m_{X}}, \\
& G_{1,1}^{3,3}=2 \cos \rho \frac{\hbar^{2}}{m_{X}} .
\end{aligned}
$$

The rotational, non-singular factors $(\alpha, \beta=x, y)$ are given by

$$
\begin{aligned}
G_{0,2}^{x, x} & =G_{2,0}^{x, x}=\frac{\hbar^{2}}{\mu_{x y}} \frac{1}{4 \cos ^{2} \rho / 2}, \\
G_{1,1}^{x, x} & =-\frac{\hbar^{2}}{m_{X}} \frac{1}{2 \cos ^{2} \rho / 2}, \\
G_{2,0}^{y, y} & =G_{0,2}^{y, y}=\frac{\hbar^{2}}{4} \frac{1}{\mu_{X Y}}, \\
G_{1,1}^{y, y} & =-\frac{\hbar^{2}}{m_{X}} \frac{\cos \rho}{2} .
\end{aligned}
$$

The Coriolis factors $(\lambda=1,2,3, \alpha=y)$ are given by

$$
\begin{aligned}
& G_{1,0}^{1, y}=-G_{0,1}^{2, y}=\hbar^{2} \frac{\sin \rho}{2 m_{X}}, \\
& G_{2,0}^{3, y}=-G_{0,2}^{3, y}=\frac{\hbar^{2}}{2 \mu_{X Y}} .
\end{aligned}
$$

The singular terms $G_{x, z}, G_{z, x}$, and $G_{z, z}$ require special care. In principle, the basis set functions $\psi_{n}^{(l)}$ in Eq. (39) include the necessary counter-terms to resolve the corresponding singularities at $\rho=0$ exactly; however, any numerical evaluations of the fractions 


$$
\frac{\rho}{\sin \rho} \text { and } \frac{\rho^{2}}{\sin ^{2} \rho}
$$

must be avoided at very small values of $\rho$ as they lead to numerical instabilities. We therefore include factors $\rho$ or $\rho^{2}$ into $G_{x, z}, G_{z, x}$, and $G_{z, z}$ explicitly, by transferring them from the basis functions $\psi_{n}^{(l)}$,

$$
\tilde{G}_{z, z}=\rho^{2} G_{z, z}, \quad \tilde{G}_{x, z}=\rho G_{x, z}, \quad \tilde{G}_{z, x}=\rho G_{z, x} .
$$

The corresponding expansion terms $\tilde{G}_{k, m}^{x, z}, \tilde{G}_{k, m}^{x, z}$, and $\tilde{G}_{k, m}^{z, z}$ are then given by

$$
\begin{aligned}
& \tilde{G}_{0,2}^{x, z}=\tilde{G}_{0,2}^{z, x}=-\tilde{G}_{2,0}^{x, z}=-\tilde{G}_{2,0}^{z, x}=\frac{\hbar^{2}}{2} \frac{1}{\mu_{X Y}} \frac{\rho}{\sin (\rho)}, \\
& \tilde{G}_{2,0}^{z, z}=\tilde{G}_{0,2}^{z, z}=\frac{\hbar^{2}}{\mu_{X Y}} \frac{\rho^{2} \cos ^{2}(\rho / 2)}{\sin ^{2}(\rho)}, \\
& \tilde{G}_{1,1}^{z, z}=\frac{2 \hbar^{2}}{m_{X}} \frac{\rho^{2} \cos ^{2}(\rho / 2)}{\sin ^{2}(\rho)} .
\end{aligned}
$$

For very small values of $\rho$ (typically $\rho<0.01 \mathrm{rad}$ ), $\tilde{G}_{\alpha, \beta}$ are expanded around $\rho=0$ to fourth order.

The pseudo-potential factors are also represented as a sum-ofproduct expansion around the non-rigid reference configuration in a similar fashion,

$$
\tilde{U}\left(r_{1}, r_{2}, \rho\right)=\sum_{k, m=0,1,2} \tilde{U}_{k, m}(\rho) f_{k}\left(r_{1}\right) f_{m}\left(r_{2}\right),
$$

with the expansion $\rho$-dependent terms given by

$$
\begin{aligned}
& \tilde{U}_{0,2}(\rho)=\tilde{U}_{2,0}(\rho)=\frac{\hbar^{2}}{8 \mu_{X Y}}\left[\frac{1}{\rho}-\frac{\rho}{\sin ^{2} \rho}-\rho\right], \\
& \tilde{U}_{1,1}(\rho)=\frac{\hbar^{2}}{4} \frac{1}{m_{X}}\left[\cos \rho\left(\frac{1}{\rho}-\frac{\rho}{\sin ^{2} \rho}+\rho\right)+2 \sin \rho\right]
\end{aligned}
$$

that are not singular at $\rho=0$,

$$
\begin{aligned}
& \tilde{U}_{0,2}(\rho)=\tilde{U}_{2,0}(\rho) \approx-\frac{\hbar^{2}}{6 \mu_{X Y}} \rho+O\left(\rho^{3}\right), \\
& \tilde{U}_{1,1}(\rho) \approx \frac{2 \hbar^{2}}{3 m_{X}} \rho+O\left(\rho^{3}\right) .
\end{aligned}
$$

These factors are also replaced by their Taylor expansion in $\rho$ of fourth order in the vicinity of $\rho=0$.

Some of the KEO expansion factors $\tilde{G}_{n, m}^{\alpha, \beta}$ and $\tilde{U}_{n, m}(\rho)$ from Eqs. (64)-(69) generated for the $\mathrm{CO}_{2}$ molecule are illustrated in Fig. 4, where PES by Huang et al. ${ }^{37}$ was used. The details of the TROVE calculations can be found in the work of Yurchenko et al. ${ }^{38}$

\section{F. Hybrid basis}

As our final basis set example for the $\mathrm{XY}_{2}$ system, we combine the advantages of the weight functions $\sin ^{l+1 / 2}(\rho)$ and $e^{-a \rho^{2} / 2}$ in the Legendre and Laguerre-based basis sets, respectively, see Eqs. (17)
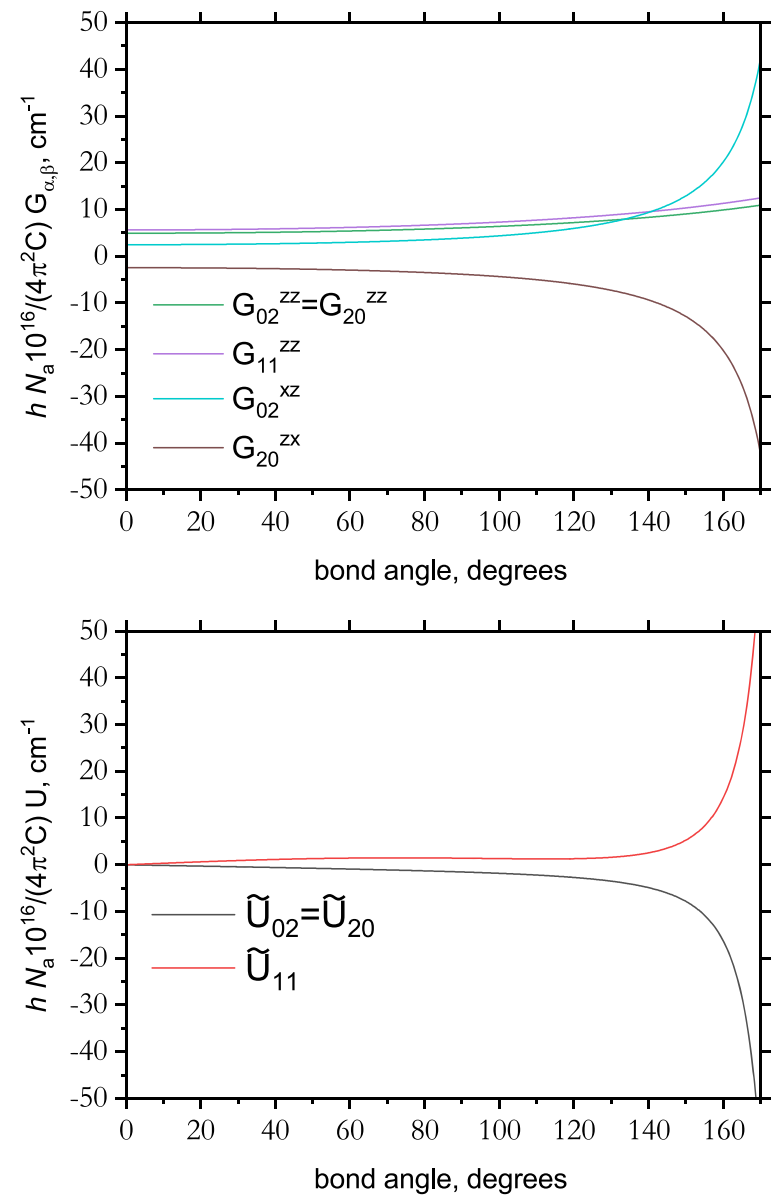

FIG. 4. The $\mathrm{KEO}$ terms for $\mathrm{CO}_{2}$.

and (39), by introducing hybrid, ortho-normal basis functions as given by

$$
\tilde{\psi}_{n}^{(l)}(\rho)=\sin ^{l+1 / 2}(\rho) \tilde{L}_{n}^{(l)}\left(a \rho^{2}\right) e^{-a \rho^{2} / 2} .
$$

Here, $\tilde{L}_{n}^{(l)}\left(a \rho^{2}\right)\left(\rho=\left[0, \ldots, 180^{\circ}\right]\right)$ are orthogonal polynomials with the weight function $\sin ^{l+1 / 2}(\rho) e^{-a \rho^{2} / 2}$. These polynomials can be numerically constructed from the original associated Laguerre polynomials from Eq. (39) (which are not orthogonal for this weight factor) and then ortho-normalized using one of the standard techniques, such as Gram-Schmidt's orthonormalization given in Eqs. (36)-(38). The correct weight $\sin ^{l+1 / 2}(\rho)$ makes these polynomials compatible with the integrals from Eqs. (25)-(35) and lead to a more compact form of the KEO. At the same time, the corresponding basis functions are more compact due to the damping term $e^{-a \rho^{2} / 2}$. The integration limit can be extended to $\rho_{\max }=180^{\circ}$, where all the KEO terms are now well defined except $G_{x, x}$. The divergence of the latter is also naturally resolved by the damping term $e^{-a \rho^{2} / 2}$.

Our tests show that in case of $\mathrm{CO}_{2}$, the hybrid basis functions $\tilde{\psi}_{n}^{(l)}$ in Eq. (72) are very similar to the Laguerre-based wavefunctions 
$\psi_{n}^{(l)}$ from Eq. (39), illustrated in Figs. 2 and 3, and therefore are not shown here.

Our hybrid basis set is an example of typical orthogonal polynomials $p_{i}(x)$ with a non-classical weight $W(x)$ constructed using a known set of orthogonal polynomials to improve the stability of orthogonalization. ${ }^{39}$ This approach can be easily further explored depending on the physical problem. For example, the isomerization of $\mathrm{HCN} / \mathrm{HNC}$ (not considered here) can be treated variationally using the following non-classical, double Gaussian weight function with a $\sin ^{l+1 / 2}$ factor:

$$
W(\rho)=\sin ^{l+1 / 2}(\rho)\left[e^{-a_{1} \rho^{2} / 2}+e^{-a_{2}(\pi-\rho)^{2} / 2}\right],
$$

where $a_{1}$ and $a_{2}$ are the corresponding structural constants defined according to Eq. (48) at $\rho=0^{\circ}$ and $\rho=180^{\circ}$, respectively.

\section{ASYMMETRIC TRIATOMIC MOLECULES YXZ}

For a non-symmetric YXZ-type triatomic, we use an embedding with the $z$ molecular frame axis oriented along the XY bond and the $y$ axis orthogonal to the molecular plane (the so-called " $x x y$ gauge" ${ }^{12}$ or $r_{1}$ embedding), as shown in Fig. 5. As mentioned before, the vibrational coordinates are $r_{1}$ (X-Y bond length), $r_{2}$ (Y-Z bond length), and $\rho=180^{\circ}-\alpha$, where $\alpha$ is the inter-bond angle $\angle \mathrm{YXZ}$. The KEO for this choice is given, e.g., by Sutcliffe and Tennyson. The methodology presented for an $\mathrm{XY}_{2}$ molecule can be directly applied to the non-symmetric case. We start by representing the KEO factors as sum-of-products using the expansion in Eq. (50). The vibrational expansion factors $G_{k, m}^{\lambda, \lambda^{\prime}}\left(\lambda, \lambda^{\prime}=1,2,3\right)$ are then given by

$$
\begin{aligned}
G_{0,0}^{1,1} & =G_{2,0}^{3,3}=\frac{\hbar^{2}}{\mu_{X Y}}, \\
G_{0,0}^{1,2} & =G_{0,0}^{2,1}=-\cos \rho \frac{\hbar^{2}}{m_{X}}, \\
G_{0,1}^{1,3} & =G_{1,0}^{2,3}=G_{0,1}^{3,1}=G_{1,0}^{3,2}=\sin \rho \frac{\hbar^{2}}{m_{X}}, \\
G_{0,0}^{2,2} & =G_{0,2}^{3,3}=\frac{\hbar^{2}}{\mu_{X Z}}, \\
G_{1,1}^{3,3} & =2 \cos \rho \frac{\hbar^{2}}{m_{X}}
\end{aligned}
$$

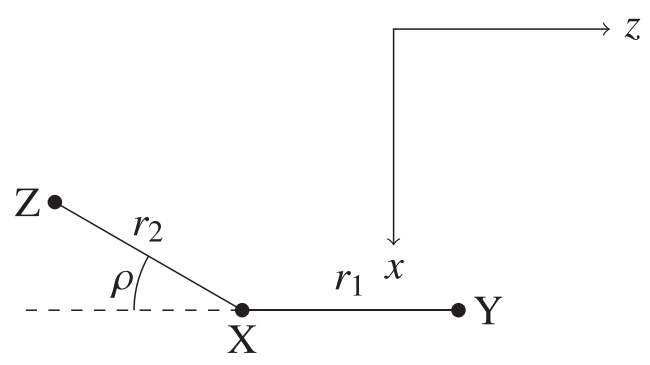

FIG. 5. The orientation of the molecular frame axes for the asymmetric molecule in the $r_{1}$ embedding with the $Z$ axis parallel to the $Y-X$ bond with the bond length $r_{1}$. The second bond $Z-X$ is with the length $r_{2}$. For clarity, the position of the axes is not at the center of mass of the molecule. where

$$
\frac{1}{\mu_{X Y}}=\frac{m_{X}+m_{Y}}{m_{X} m_{Y}} \text { and } \frac{1}{\mu_{X Z}}=\frac{m_{X}+m_{Z}}{m_{X} m_{Y}} .
$$

The non-zero rotational factors $G_{n, m}^{\alpha, \beta}(\alpha, \beta=x, y)$ and Coriolis factors $G_{n, m}^{\lambda, \alpha}(\lambda=1,2,3, \alpha=y)$ are given by

$$
\begin{gathered}
G_{2,0}^{x, x}=G_{2,0}^{y, y}=\frac{\hbar^{2}}{\mu_{X Y}}, \\
G_{1,1}^{x, z}=G_{1,1}^{z, x}=\hbar^{2} \frac{\rho}{\sin \rho m_{X}}, \quad G_{2,0}^{x, z}=G_{2,0}^{z, x}=\hbar^{2} \frac{\rho \cos \rho}{\sin \rho \mu_{X Y}}, \\
G_{0,2}^{z, z}=\hbar^{2} \frac{\rho^{2}}{\sin ^{2} \rho \mu_{X Z}}, G_{1,1}^{z, z}=\hbar^{2} \frac{2 \rho^{2} \cos \rho}{\sin ^{2} \rho m_{X}}, G_{2,0}^{z, z}=\hbar^{2} \frac{\rho^{2} \cos ^{2} \rho}{\sin ^{2} \rho \mu_{X Y}}, \\
G_{1,0}^{2, y}=-\hbar^{2} \frac{\sin \rho}{m_{X}}, G_{1,1}^{3, y}=-\hbar^{2} \frac{\cos \rho}{m_{X}}, G_{2,0}^{3, y}=-\frac{\hbar^{2}}{\mu_{X Y}} .
\end{gathered}
$$

The treatment of the singular term $U$, also expanded according to Eq. (67), depends on whether $\sqrt{\sin \rho}$ or $\sqrt{\rho}$ is used. For the $\sqrt{\sin \rho}$ choice (Legendre polynomials), the pseudo-potential function $\tilde{U}$ coincides with that of Eq. (19), and thus, the only non-zero term is

$$
\tilde{U}=\hbar^{2} \frac{\sin (2 \rho)}{2 r_{1} r_{2} m_{X}}, \quad \text { with } \quad \tilde{U}_{1,1}(\rho)=\hbar^{2} \frac{\sin (2 \rho)}{2 m_{X}} .
$$

The pseudo-potential expansion factors for the $\sqrt{\rho}$ choice used for the Laguerre-type function are given by

$$
\begin{aligned}
& \tilde{U}_{2,0}(\rho)=\frac{\hbar^{2}}{8 \mu_{X Y}} \frac{1}{\sin ^{2}(\rho)}\left[\frac{\sin ^{2} \rho}{\rho}+\rho\left(\cos ^{2}(\rho)-2\right)\right], \\
& \tilde{U}_{1,1}(\rho)=\frac{\hbar^{2}}{4 m_{X}} \frac{1}{\sin ^{2}(\rho)}\left[-\rho \cos ^{3}(\rho)+2 \sin ^{3}(\rho)+\frac{\cos \rho}{\rho}\right], \\
& \tilde{U}_{0,2}(\rho)=\frac{\hbar^{2}}{8 \mu_{X Z}} \frac{1}{\sin ^{2}(\rho)}\left[\frac{\sin ^{2} \rho}{\rho}+\rho\left(\cos ^{2}(\rho)-2\right)\right],
\end{aligned}
$$

none of which is singular,

$$
\begin{aligned}
& \tilde{U}_{2,0}(\rho) \approx-\frac{\hbar^{2}}{6} \frac{1}{\mu_{X Y}} \rho+O\left(\rho^{3}\right), \\
& \tilde{U}_{1,1}(\rho) \approx \frac{2 \hbar^{2}}{3} \frac{1}{m_{X}} \rho+O\left(\rho^{3}\right), \\
& \tilde{U}_{0,2}(\rho) \approx-\frac{\hbar^{2}}{6} \frac{1}{\mu_{X Z}} \rho+O\left(\rho^{3}\right) .
\end{aligned}
$$

For very small values of $\rho$, both $\tilde{G}_{\alpha, \beta}$ and $\tilde{U}$ are replaced by their Taylor expansion in $\rho$ of fourth order when applied to the region of small values of $\rho$. 
Analogously to the $\mathrm{XY}_{2}$ treatment above, the integrals in Eqs. (26)-(35) are used.

\section{RO-VIBRATIONAL TROVE}

The KEO and the associated Legendre and Laguerre polynomials based basis set presented above have been implemented in the variational program TROVE. ${ }^{1}$ TROVE solves the ro-vibrational Schrödinger equation using a multi-layer contraction scheme (see, for example, the work of Yurchenko, Yachmenev, and Ovsyannikov $\left.{ }^{4}\right)$. At step 1 , the $1 \mathrm{D}$ primitive basis set functions $\Phi_{v_{1}}\left(r_{1}\right)$, $\Phi_{v_{2}}\left(r_{2}\right)$ (stretching), and $\Phi_{v_{3}}^{(l)}(\rho)$ (bending) are obtained by numerically solving the corresponding Schrödinger equations. A $1 D$ Hamiltonian operator for a given mode is constructed by setting all other degrees of freedom to their equilibrium values. The two equivalent stretching equations are solved on a grid of about 1000 points using the Numerov-Cooley approach, ${ }^{40,41}$ with the grid values of $r_{1}$ and $r_{2}$ ranging typically from $r_{\mathrm{e}}-0.5 \AA$ to $r_{\mathrm{e}}+1.0 \AA$. These stretching basis functions are then used to compute $1 \mathrm{D}$ matrix elements of $1 / r_{i}, 1 / r_{i}^{2}$ from the expansions of $G_{\lambda, \mu}$ and $U$ in Eqs. (54)-(63) and Eqs. (68) and (69) as well as of all other 1D combinations of $r_{i}$ used to represent a potential energy function. The latter is assumed to be given in a sum-of-product form as well, e.g.,

$$
V\left(r_{1}, r_{2}, \rho\right)=\sum_{i, j} f_{i, j}(\rho) y_{1}^{i} y_{2}^{j}
$$

where

$$
\begin{aligned}
y_{1} & =1-\exp \left[-a_{1}\left(r_{1}-r_{1}^{\mathrm{e}}\right)\right], \\
y_{2} & =1-\exp \left[-a_{2}\left(r_{2}-r_{2}^{\mathrm{e}}\right)\right], \\
f_{i, j}(\rho) & =\sum_{k} f_{i, j, k}\left(\cos (\rho)-\cos \left(\rho_{\mathrm{e}}\right)\right)^{k}
\end{aligned}
$$

with $r_{1}^{\mathrm{e}}, r_{2}^{\mathrm{e}}$, and $\rho_{\mathrm{e}}$ as the corresponding equilibrium values.

The bending mode wavefunctions $\Phi_{v_{3}}^{(l)}(\rho)$ are obtained as solutions of a 1D Schrödinger equation for the Hamiltonian operator in Eq. (47) using the Laguerre-polynomial basis set $\psi_{n}^{(l)}(\rho)$ from Eq. (39). Here, $l$ is a parameter ranging from 0 to $l_{\max }$ where $l_{\max }$ $\leq J_{\max }$. The $2 \mathrm{D}$ index $n, l$ is combined into a $1 \mathrm{D}$ index $v_{3}$ as

$$
v_{3}^{l}=n \times\left(l_{\max }+1\right)+l .
$$

The matrix elements of all bending properties in the KEO on $\left|v_{3}^{l}\right\rangle=\Phi_{v_{3}}^{(l)}(\rho)$ are obtained numerically on a grid of $\sim 1000$ points using quadruple precision. When computing the matrix elements of the $\rho$-dependent factors $G_{\lambda, \lambda^{\prime}}$, the pseudo-potential matrix elements $\left\langle v_{3}^{l}\left|\tilde{U}_{m, n}\right| v_{3}^{\prime l}\right\rangle$ and $\left\langle v_{3}^{l}\left|\tilde{G}_{m, n}^{z, z}\right| v_{3}^{\prime l}\right\rangle$ are combined with the matrix element $\left\langle v_{3}^{l}\left|\frac{\partial}{\partial \rho} G_{m, n}^{3,3} \frac{\partial}{\partial \rho}\right| v_{3}^{\prime l}\right\rangle$ into one term as [see Eq. (27)]

$$
\begin{aligned}
G_{v_{3}, v_{3}^{\prime}, l}^{3,3, m . n} & \equiv-\left\langle v_{3}^{l}\left|\frac{\partial}{\partial \rho} G_{m, n}^{3,3} \frac{\partial}{\partial \rho}\right| v_{3}^{\prime l}\right\rangle+\frac{2}{\hbar^{2}}\left\langle v_{3}^{l}\left|U_{m, n}\right| v_{3}^{\prime l}\right\rangle+l^{2}\left\langle v_{3}^{l}\left|G_{m, n}^{z, z}\right| v_{3}^{\prime l}\right\rangle \\
= & \int_{0}^{\pi}\left[\sin \rho \frac{\partial \phi_{n}^{(l)}}{\partial \rho} G_{m, n}^{3,3} \frac{\partial \phi_{n^{\prime}}^{(l)}}{\partial \rho}+\frac{2}{\hbar^{2}} \phi_{n}^{(l)} \tilde{U}_{m, n} \phi_{n^{\prime}}^{(l)}\right. \\
& \left.+l^{2} \sin \rho \chi_{n}^{(l)} \tilde{G}_{n, m}^{z, z} \chi_{n^{\prime}}^{(l)}\right] d \rho
\end{aligned}
$$

Thus, the rotational KEO term $G_{z, z} J_{z}^{2}$ is fully accounted for already at this stage. Due to the constraint between the vibrational parameter $l$ and the rotational quantum number $k, l=|k|$, most of the matrix elements of the Hamiltonian components are diagonal in $k$, except those containing $\hat{J}_{x}^{2}, \hat{J}_{y}^{2}$, and $\hat{J}_{x} \hat{J}_{z}$. Therefore, most of the bending matrix elements for $\Phi_{v_{3}}^{(l)}$ are computed for $\Delta l=0$ only, except for $G_{z x}, G_{x z}(\Delta l= \pm 1)$ and $G_{x, x}, G_{y, y}(\Delta l= \pm 2)$.

In the case of a symmetric molecule $\mathrm{XY}_{2}$, at step 2, two reduced problems for the 2D stretching and $1 \mathrm{D}$ bending reduced Hamiltonians are solved variationally on the primitive basis sets introduced above, with two equivalent modes $r_{1}$ and $r_{2}$. The reduced Hamiltonian operators are constructed by averaging the $3 \mathrm{D}$ vibrational $(J=0)$ Hamiltonian over the ground state basis functions as follows:

$$
\begin{aligned}
\hat{H}_{\text {str }}^{(1)}\left(r_{1}, r_{2}\right) & =\left\langle 0_{3}^{0}\left|\hat{H}^{3 \mathrm{D}}\right| 0_{3}^{0}\right\rangle, \\
\hat{H}_{\text {bnd }}^{(2)}(\rho) & =\left\langle 0_{1}\left|\left\langle 0_{2}\left|\hat{H}^{3 \mathrm{D}}\right| 0_{2}\right\rangle\right| 0_{1}\right\rangle,
\end{aligned}
$$

where $\left|v_{i}\right\rangle=\Phi_{v_{1}}\left(r_{1}\right) \Phi_{v_{2}}\left(r_{2}\right)(i=1,2)$ are stretching and $\left|v_{3}^{l}\right\rangle$ $=\Phi_{v_{3}}^{(l)}(\rho)$ are bending vibrational basis functions. In principle, the Hamiltonian matrix $v_{3}^{\max } \times v_{3}^{\max }$

$$
H_{v_{3}, v_{3}^{\prime}}^{(2), l}=\left\langle v_{3}^{l}\left|\hat{H}_{\text {bnd }}^{(2)}(\rho)\right| v_{3}^{\prime l}\right\rangle
$$

is block-diagonal in $l$; for a technical reason, this is currently not recognized, and the whole matrix is diagonalized, which is not a problem as the dimension $\left(v_{3}^{\max }+1\right)$ is relatively small.

The eigenfunctions of $\hat{H}_{\mathrm{str}}^{(1)}$ and $\hat{H}_{\mathrm{str}}^{(2)}, \Psi_{i_{1}}^{(1)}\left(r_{1}, r_{2}\right)$ and $\Psi_{i_{2}, l}^{(2)}(\rho)$, respectively, are then symmetrized using the automatic symmetryadaptation technique, ${ }^{4}$ which is an important part of step 2, in this case using the $\mathcal{C}_{2 \mathrm{v}}(\mathrm{M})$ molecular symmetry group.

Step 3 involves an eigensolution of the $J=0$ Hamiltonian on a $3 \mathrm{D}$ vibrational basis set formed as symmetry-adapted products of $\Psi_{i_{1}}^{(1)}\left(r_{1}, r_{2}\right)$ and $\Psi_{i_{2}, l}^{(2)}(\rho)$,

$$
\Psi_{i_{1}, i_{2}, l}^{\Gamma_{\mathrm{vib}}}=\left\{\Psi_{i_{1}}^{(1)}\left(r_{1}, r_{2}\right) \Psi_{i_{2}, l}^{(2)}(\rho)\right\}^{\Gamma_{\mathrm{vib}}},
$$

where $\Gamma_{\text {vib }}$ is the vibrational symmetry in the $\mathcal{C}_{2 \mathrm{v}}(\mathrm{M})$ molecular group symmetry used to classify the irreducible representations (irreps) of the ro-vibrational states of $\mathrm{XY}_{2} \cdot \mathcal{C}_{2 \mathrm{v}}(\mathrm{M})$ is comprised of four irreps $A_{1}, A_{2}, B_{1}$, and $B_{2}$. The ro-vibrational basis set is then constructed as a symmetrized product of the vibrational eigenfunctions of the $J=0$ problem and rigid rotor wavefunctions.

In the case of an asymmetric YXZ-type triatomic, at step 2, all three modes are processed independently as $1 \mathrm{D}$ reduced Hamiltonians. The vibrational eigensolutions are formally symmetrized according to the representations of the $\mathcal{C}_{\mathrm{s}}(\mathrm{M})$ molecular symmetry group, which in this case can be only of $A^{\prime}$, so are the vibrational $(J=0)$ basis set functions

$$
\Psi_{i_{1}, i_{2}, i_{3}, l}^{A^{\prime}}=\Psi_{i_{1}}^{(1)}\left(r_{1}\right) \Psi_{i_{2}}^{(2)}\left(r_{2}\right) \Psi_{i_{3}, l}^{(3)}(\rho) .
$$

The ro-vibrational basis functions transform according to $A^{\prime}$ or $A^{\prime \prime}$ of $\mathcal{C}_{\mathrm{s}}(\mathrm{M})$. 


\section{COMPUTATIONAL EXAMPLES}

\section{A. Symmetric molecule: Computing hot $\mathrm{CO}_{2}$ spectra}

Here, we present an example of the computation of spectra for a linear triatomic molecule $\mathrm{CO}_{2}$. The PES Ames-2016 by Huang et al. $^{37}$ was used. For the kinetic energy factors given by Eqs. (54)(68), the non-rigid configuration consisted of 3000 equidistant grid points ranging from $0^{\circ}$ to $170^{\circ}$. The Laguerre basis set was generated for $l_{\max }=40$. The nuclear masses of $\mathrm{C}$ and $\mathrm{O}$ were used. The stretching and bending primitive basis set functions ranged up to 56 and 64 quanta, respectively. The stretching basis set was generated by solving a 1D Schrödinger equation using the Numerov-Cooley procedure on an equidistant grid of 1000 points ranging from $r_{\mathrm{e}}-$ $0.4 \AA$ to $r_{\mathrm{e}}+1.0 \AA$ with $r_{\mathrm{e}}=1.1613997 \AA$. The potential energy function Ames-2016 was re-expanded into a 12th order Taylor-type expansion in terms of the coordinates $y_{1}$ and $y_{2}$ from Eqs. (89) and (90) with $a_{1}=a_{2}=1 / \AA ̊$. After solving the reduced problems given by Eqs. (93) and (94), a 3D vibrational basis set was constructed from symmetry-adapted $\left[\mathcal{C}_{2 \mathrm{v}}(\mathrm{M})\right]$ combinations, Eq. (95), corresponding to the energies below $h c \times 40000 \mathrm{~cm}^{-1}$. The $J=0$ energies were computed by solving the vibrational Schrödinger equation variationally. Eigenfunctions of the $J=0$ problem corresponding to states below $32000 \mathrm{~cm}^{-1}$ were used to build the ro-vibrational basis set with the rotational part as rigid rotor oscillators, after being symmetrized according to $\mathcal{C}_{2 \mathrm{v}}(\mathrm{M})$. The parameter $a$ in the definition of the Laguerre polynomials in Eq. (39) was set to 58.5354 according to Eq. (48).
To compute all ro-vibrational energies for $J \leq 230$ with this basis set, it took less than $24 \mathrm{~h}$ on the Intel Xeon Skylake $2.6 \mathrm{GHz} 32$ cores per node systems available via the DIRAC project.

Some of the energies of $\mathrm{CO}_{2}(J=0,1)$ computed with TROVE are compared to the energies computed using the DVR3D program $^{18}$ in Table I using the same potential energy surface, which show a close agreement within $0.03 \mathrm{~cm}^{-1}$.

Figure 6 (left display) shows how ro-vibrational residuals depend on the rotational quantum number $J$ for the ground vibrational states and the fundamentals $(J=0, \ldots, 40)$. The same figure (right display) shows a similar comparison with the experimentally derived ro-vibrational energies of $\mathrm{CO}_{2}$ from the HITRAN database $^{43}$ with a larger $J$ range $(J \leq 128)$. Here, band center shifts were applied to the TROVE term values to match the HITRAN energies (see the work of Yurchenko et al. ${ }^{38}$ for details). The complete set of energies for $J \leq 230$ can be found in the work of Yurchenko et al.

\section{B. Asymmetric molecule: $\mathrm{HCN}$ term values}

As an example of applications of the presented methodology to YXZ molecules, we use the spectroscopic model developed recently for HCN by Makhnev et al. ${ }^{46}$ Here, we have used their empirical PES to compute ro-vibrational energies of $\mathrm{H}^{12} \mathrm{C}^{14} \mathrm{~N}$ and compare to energies reported by Makhnev et al. ${ }^{46}$ To this end, the KEO represented by Eqs. (73)-(88) was used with the molecular $z$ axis placed parallel to the $\mathrm{C}-\mathrm{N}$ bond. The PES was re-expanded into a sixth

TABLE I. $\mathrm{CO}_{2}$ term values $\left(\mathrm{cm}^{-1}\right)$ computed using Ames-2 PES with DVR3D (D) ${ }^{44}$ and TROVE $(\mathrm{T})$ from this work for $\mathrm{J}=0,1$. The HITRAN quantum numbers ${ }^{45}$ are used to assign the $\mathrm{CO}_{2}$ ro-vibrational states.

\begin{tabular}{|c|c|c|c|c|c|c|c|c|c|c|c|c|c|c|c|c|c|}
\hline \multicolumn{6}{|c|}{ Quantum numbers } & \multicolumn{3}{|c|}{ Term values $\left(\mathrm{cm}^{-1}\right)$} & \multicolumn{6}{|c|}{ Quantum numbers } & \multicolumn{3}{|c|}{ Term values $\left(\mathrm{cm}^{-1}\right)$} \\
\hline$J$ & $n_{1}$ & $n_{2}^{\operatorname{lin}}$ & $l_{2}$ & $n_{3}$ & $r$ & DVR3D & TROVE & $\mathrm{D}-\mathrm{T}$ & $J$ & $n_{1}$ & $n_{2}^{\operatorname{lin}}$ & $l_{2}$ & $n_{3}$ & $r$ & DVR3D & TROVE & $\mathrm{D}-\mathrm{T}$ \\
\hline 0 & 0 & 0 & 0 & 0 & 1 & 0.0000 & 0.0000 & 0.0000 & 1 & 0 & 1 & 1 & 0 & 1 & 668.1591 & 668.1463 & 0.0128 \\
\hline 0 & 1 & 0 & 0 & 0 & 2 & 1285.3982 & 1285.4037 & -0.0055 & 1 & 1 & 1 & 1 & 0 & 2 & 1933.2289 & 1933.2126 & 0.0163 \\
\hline 0 & 1 & 0 & 0 & 0 & 1 & 1388.2030 & 1388.2091 & -0.0061 & 1 & 1 & 1 & 1 & 0 & 1 & 2077.6410 & 2077.6241 & 0.0169 \\
\hline 0 & 2 & 0 & 0 & 0 & 3 & 2548.3302 & 2548.3428 & -0.0126 & 1 & 0 & 0 & 0 & 1 & 1 & 2349.9489 & 2349.9478 & 0.0011 \\
\hline 0 & 2 & 0 & 0 & 0 & 2 & 2671.1369 & 2671.1435 & -0.0066 & 1 & 0 & 1 & 1 & 1 & 1 & 3004.8580 & 3004.8445 & 0.0135 \\
\hline 0 & 2 & 0 & 0 & 0 & 1 & 2797.1419 & 2797.1568 & -0.0149 & 1 & 2 & 1 & 1 & 0 & 3 & 3182.2035 & 3182.1833 & 0.0202 \\
\hline 0 & 3 & 0 & 0 & 0 & 4 & 3792.6360 & 3792.6556 & -0.0196 & 1 & 2 & 1 & 1 & 0 & 2 & 3340.1160 & 3340.0964 & 0.0196 \\
\hline 0 & 3 & 0 & 0 & 0 & 3 & 3942.5071 & 3942.5173 & -0.0102 & 1 & 2 & 1 & 1 & 0 & 1 & 3501.4460 & 3501.4241 & 0.0219 \\
\hline 0 & 3 & 0 & 0 & 0 & 2 & 4064.2642 & 4064.2769 & -0.0127 & 1 & 1 & 0 & 0 & 1 & 2 & 3613.6619 & 3613.6660 & -0.0041 \\
\hline 0 & 3 & 0 & 0 & 0 & 1 & 4225.0865 & 4225.1107 & -0.0242 & 1 & 1 & 0 & 0 & 1 & 1 & 3715.6220 & 3715.6258 & -0.0038 \\
\hline 0 & 0 & 0 & 0 & 2 & 1 & 4673.3731 & 4673.3707 & 0.0024 & 1 & 1 & 1 & 1 & 1 & 2 & 4248.5414 & 4248.5240 & 0.0174 \\
\hline 0 & 4 & 0 & 0 & 0 & 5 & 5022.3280 & 5022.3544 & -0.0264 & 1 & 1 & 1 & 1 & 1 & 1 & 4391.4677 & 4391.4497 & 0.0180 \\
\hline 0 & 4 & 0 & 0 & 0 & 4 & 5197.2122 & 5197.2284 & -0.0162 & 1 & 3 & 1 & 1 & 0 & 4 & 4416.8958 & 4416.8714 & 0.0244 \\
\hline 0 & 4 & 0 & 0 & 0 & 3 & 5329.6257 & 5329.6361 & -0.0104 & 1 & 3 & 1 & 1 & 0 & 3 & 4591.8617 & 4591.8384 & 0.0233 \\
\hline 0 & 4 & 0 & 0 & 0 & 2 & 5475.5362 & 5475.5581 & -0.0219 & 1 & 3 & 1 & 1 & 0 & 2 & 4754.2080 & 4754.1836 & 0.0244 \\
\hline 0 & 4 & 0 & 0 & 0 & 1 & 5667.6298 & 5667.6633 & -0.0335 & 1 & 2 & 0 & 0 & 1 & 3 & 4854.4467 & 4854.4574 & -0.0107 \\
\hline 0 & 1 & 0 & 0 & 2 & 2 & 5915.2708 & 5915.2732 & -0.0024 & 1 & 3 & 1 & 1 & 0 & 1 & 4939.1496 & 4939.1221 & 0.0275 \\
\hline 0 & 1 & 0 & 0 & 2 & 1 & 6016.7629 & 6016.7640 & -0.0010 & 1 & 2 & 0 & 0 & 1 & 2 & 4978.6589 & 4978.6623 & -0.0034 \\
\hline 0 & 2 & 0 & 0 & 2 & 3 & 7133.8848 & 7133.8933 & -0.0085 & 1 & 2 & 0 & 0 & 1 & 1 & 5100.4945 & 5100.5062 & -0.0117 \\
\hline 0 & 2 & 0 & 0 & 2 & 2 & 7259.8259 & 7259.8256 & 0.0003 & 1 & 0 & 1 & 1 & 2 & 1 & 5316.5820 & 5316.5676 & 0.0144 \\
\hline 0 & 2 & 0 & 0 & 2 & 1 & 7377.7626 & 7377.7705 & -0.0079 & 1 & 2 & 1 & 1 & 1 & 3 & 5475.9102 & 5475.8883 & 0.0219 \\
\hline
\end{tabular}



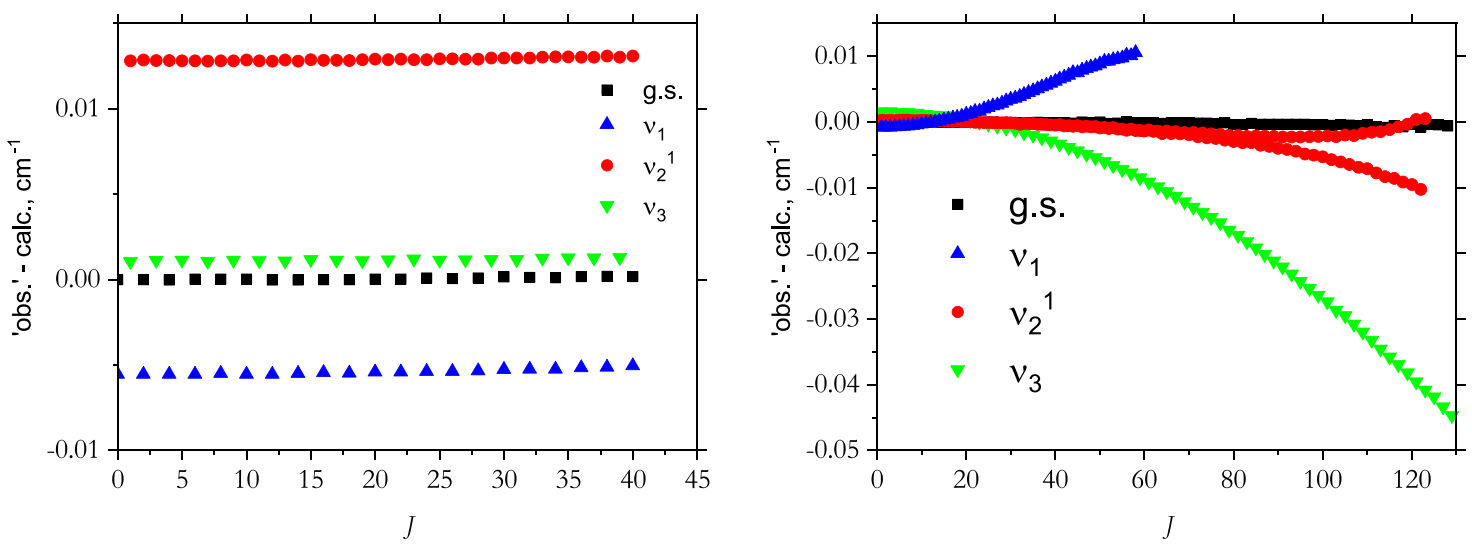

FIG. 6. Left display: The differences between the $\mathrm{CO}_{2}$ term values $\left(\mathrm{cm}^{-1}\right)$ computed using Ames-2 PES with DVR3D ${ }^{44}$ and TROVE (this work) as in Table I for three lowest vibrational states, g.s., $v_{1}, v_{2}^{1}$, and $v_{3}$ and $J=0,40$. Right display: The differences between experimentally derived ("obs.") energies of $\mathrm{CO}_{2}$ from the HITRAN database and calculated (calc.) with TROVE, where the corresponding band centers were shifted to match HITRAN, for a large range of $J$.

order Taylor-type series in terms of

$$
\begin{aligned}
& y_{1}=r_{1}-r_{1}^{\mathrm{e}}, \\
& y_{2}=r_{2}-r_{2}^{\mathrm{e}}, \\
& y_{3}=\cos (\alpha)-\cos \left(\alpha_{\mathrm{e}}\right) .
\end{aligned}
$$

The Laguerre structural parameter $a$ was set to 17.9784 according to Eq. (48). The Laguerre-type basis functions were generated on a grid of 1000 points ranging from $0^{\circ}$ to $120^{\circ}$. The basis set comprised of 48 stretching functions and 56 bending functions. The computed term values for $J=2$ are compared to the corresponding values reported by Makhnev et al. ${ }^{46}$ in Table II, showing an agreement

\begin{tabular}{|c|c|c|c|c|c|c|c|c|c|c|c|c|c|c|c|c|c|}
\hline \multicolumn{5}{|c|}{ Quantum numbers } & \multicolumn{4}{|c|}{ Term values $\left(\mathrm{cm}^{-1}\right)$} & \multicolumn{5}{|c|}{ Quantum numbers } & \multicolumn{4}{|c|}{ Term values $\left(\mathrm{cm}^{-1}\right)$} \\
\hline$v_{1}$ & $V_{2}$ & $l$ & $v_{3}$ & $e / f$ & Obs. & DVR3D & TROVE & $\mathrm{D}-\mathrm{T}$ & $v_{1}$ & $v_{2}$ & $l$ & $v_{3}$ & $e / f$ & Obs. & DVR3D & TROVE & D-T \\
\hline 0 & 0 & 0 & 0 & $e$ & 8.8692 & 8.8668 & 8.8668 & 0.0000 & 0 & 8 & 0 & 1 & $e$ & 6263.3544 & 6263.4079 & 6263.4132 & -0.0053 \\
\hline 0 & 1 & 1 & 0 & $e$ & 720.8477 & 720.8509 & 720.8488 & 0.0021 & 0 & 3 & 1 & 2 & $e$ & 6276.4258 & 6276.4331 & 6276.4296 & 0.0035 \\
\hline 0 & 2 & 0 & 0 & $e$ & 1420.3282 & 1420.3614 & 1420.3633 & -0.0019 & 0 & 6 & 2 & 1 & $e$ & 6278.4229 & 6278.4901 & 6278.4885 & 0.0016 \\
\hline 0 & 2 & 2 & 0 & $e$ & 1435.4400 & 1435.4613 & 1435.4602 & 0.0011 & 2 & 0 & 0 & 0 & $e$ & 6528.3528 & 6528.3620 & 6528.3603 & 0.0017 \\
\hline 0 & 0 & 0 & 1 & $e$ & 2105.6543 & 2105.6944 & 2105.6942 & 0.0002 & 1 & 7 & 1 & 0 & $e$ & 6718.3561 & 6718.3416 & 6718.3370 & 0.0046 \\
\hline 0 & 3 & 1 & 0 & $e$ & 2122.3417 & 2122.3805 & 2122.3773 & 0.0032 & 1 & 2 & 0 & 1 & $e$ & 6769.4994 & 6769.5340 & 6769.5351 & -0.0011 \\
\hline 0 & 7 & 1 & 0 & $e$ & 3504.0296 & 3504.0355 & 3504.0312 & 0.0043 & 1 & 2 & 2 & 1 & $e$ & 6784.1720 & 6784.1782 & 6784.1763 & 0.0019 \\
\hline 0 & 2 & 0 & 1 & $e$ & 3510.9738 & 3510.9721 & 3510.9739 & -0.0018 & 0 & 10 & 0 & 0 & $e$ & 6864.5701 & 6864.6257 & 6864.6343 & -0.0086 \\
\hline 0 & 2 & 2 & 1 & $e$ & 3525.7201 & 3525.6959 & 3525.6946 & 0.0013 & 0 & 1 & 1 & 0 & $f$ & 720.8926 & 720.8958 & 720.8937 & 0.0021 \\
\hline 1 & 1 & 1 & 0 & $e$ & 4012.9686 & 4012.9634 & 4012.9609 & 0.0025 & 0 & 2 & 2 & 0 & $f$ & 1435.4399 & 1435.4612 & 1435.4601 & 0.0011 \\
\hline 0 & 0 & 0 & 2 & $e$ & 4181.8188 & 4181.8874 & 4181.8870 & 0.0004 & 0 & 3 & 1 & 0 & $f$ & 2122.4342 & 2122.4729 & 2122.4697 & 0.0032 \\
\hline 0 & 8 & 0 & 0 & $e$ & 4183.6223 & 4183.6063 & 4183.6119 & -0.0056 & 0 & 1 & 1 & 1 & $f$ & 2814.4338 & 2814.4248 & 2814.4225 & 0.0023 \\
\hline 0 & 6 & 2 & 0 & $e$ & 4198.9831 & 4198.9695 & 4198.9681 & 0.0014 & 0 & 4 & 2 & 0 & $f$ & 2827.1335 & 2827.1630 & 2827.1618 & 0.0012 \\
\hline 0 & 3 & 1 & 1 & $e$ & 4210.0337 & 4210.0443 & 4210.0409 & 0.0034 & 0 & 7 & 1 & 0 & $f$ & 3504.1726 & 3504.1785 & 3504.1742 & 0.0043 \\
\hline 1 & 2 & 0 & 0 & $e$ & 4693.1648 & 4693.1912 & 4693.1926 & -0.0014 & 0 & 2 & 2 & 1 & $f$ & 3525.7200 & 3525.6958 & 3525.6945 & 0.0013 \\
\hline 1 & 2 & 2 & 0 & $e$ & 4708.0591 & 4708.0625 & 4708.0610 & 0.0015 & 1 & 1 & 1 & 0 & $f$ & 4013.0143 & 4013.0090 & 4013.0065 & 0.0025 \\
\hline 0 & 7 & 1 & 0 & $e$ & 4865.6296 & 4865.6041 & 4865.5987 & 0.0054 & 0 & 6 & 2 & 0 & $f$ & 4198.9825 & 4198.9689 & 4198.9675 & 0.0014 \\
\hline 0 & 1 & 1 & 2 & $e$ & 4887.0403 & 4887.0180 & 4887.0156 & 0.0024 & 0 & 2 & 2 & 2 & $f$ & 5594.8511 & 5594.7926 & 5594.7911 & 0.0015 \\
\hline 0 & 4 & 0 & 1 & $e$ & 4896.9384 & 4896.9692 & 4896.9727 & -0.0035 & 1 & 4 & 2 & 0 & $f$ & 6060.8190 & 6060.8312 & 6060.8296 & 0.0016 \\
\hline 0 & 4 & 2 & 1 & $e$ & 4911.8322 & 4911.8538 & 4911.8524 & 0.0014 & 1 & 1 & 1 & 1 & $f$ & 6092.1407 & 6092.1347 & 6092.1319 & 0.0028 \\
\hline 0 & 3 & 1 & 0 & $e$ & 5375.7074 & 5375.7355 & 5375.7320 & 0.0035 & 0 & 9 & 1 & 0 & $f$ & 6206.5672 & 6206.5780 & 6206.5717 & 0.0063 \\
\hline 1 & 0 & 0 & 1 & $e$ & 5402.4452 & 5402.4557 & 5402.4547 & 0.0010 & 0 & 3 & 1 & 2 & $f$ & 6276.5173 & 6276.5246 & 6276.5210 & 0.0036 \\
\hline 0 & 8 & 0 & 0 & $e$ & 5534.8809 & 5534.8643 & 5534.8716 & -0.0073 & 0 & 6 & 2 & 1 & $f$ & 6278.4223 & 6278.4895 & 6278.4879 & 0.0016 \\
\hline 0 & 8 & 2 & 0 & $e$ & 5550.4433 & 5550.4259 & 5550.4244 & 0.0015 & 1 & 7 & 1 & 0 & $f$ & 6718.5020 & 6718.4875 & 6718.4829 & 0.0046 \\
\hline 0 & 2 & 0 & 2 & $e$ & 5580.5247 & 5580.5002 & 5580.5017 & -0.0015 & 1 & 2 & 2 & 1 & $f$ & 6784.1719 & 6784.1781 & 6784.1762 & 0.0019 \\
\hline
\end{tabular}

TABLE II. HCN J $=2$ term values $\left(\mathrm{cm}^{-1}\right)$ computed with DVR3D (D) ${ }^{44}$ using a spectroscopically determined PES by Makhnev et al. ${ }^{46}$ and TROVE (T) from this work using the same PES. Experimental term of $\mathrm{HCN}$ by Mellau ${ }^{49}$ is given as reference. The linear molecule quantum numbers $v_{1}, v_{2}^{l}, v_{3}$, and elf are used. 
significantly better than $0.01 \mathrm{~cm}^{-1}$ for term values below $6700 \mathrm{~cm}^{-1}$ fully reproducing the $l$-type doubling splitting. These deviations can be attributed mostly to the difference in treating the potential energy surface of $\mathrm{HCN}$. $^{47,48}$

\section{CONCLUSION}

In this work, we report an implementation of the exact kinetic energy operator for triatomics in the variational program TROVE. The implementation is based on the formulation of $\mathrm{KE}$ operators developed by Carter, Handy, and Sutcliffe ${ }^{11}$ and Sutcliffe and Tennyson $^{13}$ over 30 years ago for the valence coordinates (bond lengths and inter-bond angle) using the bisector $\left(\mathrm{XY}_{2}\right)$ and the bond-vector (XYZ) embeddings. The singularity appearing in these KE operators at the linear geometry is treated by properly selecting the basis set either as associated Legendre or Laguerre polynomials, which are optimized by solving the reduced 1D problem for the bending degree of freedom and used to construct vibrational basis functions of the FBR type as sum-of-products. The exact KE operators are also represented as sum-of-products of $1 \mathrm{D}$ stretching functions $1 / r_{i}$ and $1 / r_{i}^{2}(i=1,2)$ around a non-rigid reference configuration defined by the bending angle $\rho=0, \ldots, 180$. This procedure is integrated into the TROVE computational pipeline, which has been previously optimized for efficient production of hot spectra of polyatomic molecules characterized by high rotational and vibrational excitations. In order to illustrate the accuracy of our implementation, the ro-vibrational energies of $\mathrm{CO}_{2}$ and $\mathrm{HCN}$ were computed and compared to previous DVR3D calculations based on the same potential energy surfaces, as well as to experimentally derived energies from HITRAN $\left(\mathrm{CO}_{2}\right)$, showing excellent agreement. Moreover, we have recently successfully used this methodology to produce hot line lists for $\mathrm{SiO}_{2}{ }^{50}$ and $\mathrm{CO}_{2},{ }^{38}$ covering rotational excitations up to $J=230$ and 255 , respectively, and it is being used to compute hot line lists for $\mathrm{CaOH}, \mathrm{NaOH}$, and $\mathrm{KOH}$.

TROVE is an open-access variational Fortran 2003 code. $^{5}$ The TROVE input files used in this work for $\mathrm{CO}_{2}$ and $\mathrm{HCN}$ rovibrational calculations are provided in the supplementary material and also included in the TROVE benchmark set. ${ }^{5}$

The methodology presented in this work can be straightforwardly extended to other popular coordinate systems and embeddings including the Radau coordinates and scattering coordinates.

Many larger polyatomic systems also allow for the KEO to be expressed in an exact sum-of-product form. We shall exploit this representation in future work with a methodology similar to the one presented for linear molecules. We are also planning to use the hybrid basis sets, constructed by orthogonalizing polynomials with the correct behavior at linear configurations, as described in Sec. II F, to larger molecules, starting from chain tetratomics such as $\mathrm{HCCH}$, where we can follow a methodology developed in the literature, e.g., by Bramley and Handy ${ }^{16}$ or Schiffel and Manthe. ${ }^{32}$

\section{SUPPLEMENTARY MATERIAL}

See the supplementary material for the TROVE input files specifying the spectroscopic models (PES) and the level of theory for $\mathrm{CO} 2$ and $\mathrm{HCN}$ used in this work.

\section{ACKNOWLEDGMENTS}

This work was supported by the ERC under Advanced Investigator Project No. 267219 and by STFC under Project Nos. ST/M001334/1 and ST/J002925. The authors acknowledge the use of the UCL Legion High Performance Computing Facility (Legion@UCL), and associated support services, in the completion of this work, along with the Cambridge COSMOS SMP system, part of the STFC DiRAC HPC Facility supported by BIS National E-infrastructure capital, Grant Nos. ST/J005673/1, STFC ST/H008586/1, and ST/K00333X/1.

\section{DATA AVAILABILITY}

The data that support the findings of this study are available within the article and its supplementary material.

\section{REFERENCES}

${ }^{1}$ S. N. Yurchenko, W. Thiel, and P. Jensen, J. Mol. Spectrosc. 245, 126 (2007).

${ }^{2}$ J. Tennyson and S. N. Yurchenko, Int. J. Quantum Chem. 117, 92 (2017).

${ }^{3}$ A. Yachmenev and S. N. Yurchenko, J. Chem. Phys. 143, 014105 (2015).

${ }^{4}$ S. N. Yurchenko, A. Yachmenev, and R. I. Ovsyannikov, J. Chem. Theory Comput. 13, 4368 (2017).

${ }^{\mathbf{5}}$ S. N. Yurchenko, R. J. Barber, J. Tennyson, W. Thiel, and P. Jensen, J. Mol. Spectrosc. 268, 123 (2011).

${ }^{6}$ A. Yachmenev, S. N. Yurchenko, I. Paidarová, P. Jensen, W. Thiel, and S. P. A. Sauer, J. Chem. Phys. 132, 114305 (2010).

${ }^{7}$ A. Owens and A. Yachmenev, J. Chem. Phys. 148, 124102 (2018).

${ }^{8}$ A. Campargue, S. Kassi, A. Yachmenev, A. A. Kyuberis, J. Küpper, and S. N. Yurchenko, Phys. Rev. Res. 2, 023091 (2020).

${ }^{9}$ K. L. Chubb, A. Yachmenev, J. Tennyson, and S. N. Yurchenko, J. Chem. Phys. 149, 014101 (2018).

${ }^{10}$ K. L. Chubb, J. Tennyson, and S. N. Yurchenko, Mon. Not. R. Astron. Soc. 493, 1531 (2020).

${ }^{11}$ S. Carter, N. Handy, and B. Sutcliffe, Mol. Phys. 49, 745 (1983).

${ }^{12}$ R. G. Littlejohn and M. Reinsch, Rev. Mod. Phys. 69, 213 (1997).

${ }^{13}$ B. T. Sutcliffe and J. Tennyson, Int. J. Quantum Chem. 39, 183 (1991).

${ }^{14}$ T. J. Lukka, J. Chem. Phys. 102, 3945 (1995)

${ }^{15}$ J. K. Watson, J. Mol. Spectrosc. 228, 645 (2004), Special Issue Dedicated to Dr. Jon T. Hougen on the Occasion of His 68th Birthday.

${ }^{16}$ M. J. Bramley and N. C. Handy, J. Chem. Phys. 98, 1378 (1993).

${ }^{17}$ P. Jensen, J. Mol. Spectrosc. 128, 478 (1988).

${ }^{18}$ J. Tennyson, M. A. Kostin, P. Barletta, G. J. Harris, O. L. Polyansky, J. Ramanlal, and N. F. Zobov, Comput. Phys. Commun. 163, 85 (2004).

${ }^{19}$ D. W. Schwenke, J. Chem. Phys. 142, 144107 (2015).

${ }^{20}$ I. Bohaček, D. Papoušek, Š. Pick, and V. Špirko, Chem. Phys. Lett. 42, 395 (1976).

${ }^{21}$ M. Perić and S. D. Peyerimhoff, J. Mol. Spectrosc. 212, 142 (2002).

${ }^{22}$ B. Podolsky, Phys. Rev. 32, 812 (1928).

${ }^{23}$ G. O. Sørensen, in Large Amplitude Motion in Molecules II, Topics in Current Chemistry Vol. 82 , edited by Multidisciplinary Journal of Social Diversity et al. (Springer Berlin Heidelberg, Heidelberg, 1979), pp. 97-175.

${ }^{24}$ A. Nauts and X. Chapuisat, Mol. Phys. 55, 1287 (1985).

${ }^{25}$ E. B. Wilson, J. C. Decius, and P. C. Cross, Molecular Vibrations: The Theory of Infrared and Raman Vibrational Spectra (McGraw-Hill, 1955).

${ }^{26}$ X. Chapuisat, A. Nauts, and J.-P. Brunet, Mol. Phys. 72, 1 (1991).

${ }^{27} \mathrm{~J}$. Tennyson and B. T. Sutcliffe, J. Mol. Spectrosc. 101, 71 (1983).

${ }^{28}$ H. Wei and T. Carrington, J. Chem. Phys. 101, 1343 (1994).

${ }^{29}$ P. Jensen, Comput. Phys. Rep. 1, 1 (1983). 
${ }^{30}$ M. Abramowitz and I. A. Stegun, Handbook of Mathematical Functions with Formulas, Graphs, and Mathematical Tables, ninth Dover printing, tenth GPO printing ed. (Dover, New York, 1964).

${ }^{31}$ I. S. Gradshteyn and I. M. Ryzhik, Table of Integrals, Series, and Products (Academic Press, 2014).

${ }^{32}$ G. Schiffel and U. Manthe, Chem. Phys. 374, 118 (2010).

${ }^{33}$ See https://people.sc.fsu.edu/ jburkardt/ for a Fortran subroutine for associated Laguerre polynomials.

${ }^{34}$ E. S. Medvedev, V. V. Meshkov, A. V. Stolyarov, and I. E. Gordon, J. Chem. Phys. 143, 154301 (2015).

${ }^{35}$ E. S. Medvedev, V. G. Ushakov, E. K. Conway, A. Upadhyay, I. E. Gordon, and J. Tennyson, J. Quant. Spectrosc. Radiat. Transfer 252, 107084 (2020).

${ }^{36}$ J. T. Hougen, P. R. Bunker, and J. W. C. Johns, J. Mol. Spectrosc. 34, 136 (1970).

${ }^{37}$ X. Huang, D. W. Schwenke, R. S. Freedman, and T. J. Lee, J. Quant. Spectrosc. Radiat. Transfer 203, 224 (2017)

${ }^{38}$ S. N. Yurchenko, T. M. Mellor, R. S. Freedman, and J. Tennyson, Mon. Not. R. Astron. Soc. 496, 5282 (2020).

${ }^{39}$ W. H. Press and S. A. Teukolsky, Comput. Phys. 4, 423 (1990).

${ }^{40}$ B. V. Noumerov, Mon. Not. R. Astron. Soc. 84, 592 (1924).

${ }^{41}$ J. W. Cooley, Math. Comput. 15, 363 (1961).
${ }^{42}$ P. R. Bunker and P. Jensen, Molecular Symmetry and Spectroscopy, 2nd ed. (NRC Research Press, Ottawa, 1998).

${ }^{43}$ I. E. Gordon et al., J. Quant. Spectrosc. Radiat. Transfer 203, 3 (2017).

${ }^{44}$ E. J. Zak, J. Tennyson, O. L. Polyansky, L. Lodi, S. A. Tashkun, and V. I. Perevalov, J. Quant. Spectrosc. Radiat. Transfer 177, 31 (2016).

${ }^{45}$ L. S. Rothman and L. D. Young, J. Quant. Spectrosc. Radiat. Transfer 25, 505 (1981).

${ }^{46}$ V. Y. Makhnev, A. A. Kyuberis, O. L. Polyansky, I. I. Mizus, J. Tennyson, and N. F. Zobov, J. Mol. Spectrosc. 353, 40 (2018).

${ }^{47}$ D. Papoušek and M. R. Aliev, Molecular Vibrational-Rotational Spectra (Elsevier, Amsterdam, 1982)

${ }^{48}$ A. G. Maki, G. C. Mellau, S. Klee, M. Winnewisser, and W. Quapp, J. Mol. Spectrosc. 202, 67 (2000).

${ }^{49}$ G. C. Mellau, J. Chem. Phys. 134, 234303 (2011).

${ }^{50}$ V. H. Clark, A. Owens, J. Tennyson, and S. N. Yurchenko, J. Quant. Spectrosc. Radiat. Transfer 246, 106929 (2020).

${ }^{51}$ See https://github.com/Trovemaster/TROVE for the Fortran package TROVE.

${ }^{\mathbf{5 2}}$ See https://github.com/Trovemaster/TROVE-benchmarks for a set of examples of input files for computing energies and intensities of polyatomic molecules using the variational program TROVE. 